\title{
\#3-Alaska-1903 $D^{2}=4246 * 4276$ H. $4763-4778$ \\ July 1I-Auk. 6, 1903
}




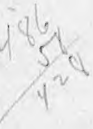

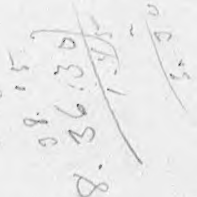

$$
\begin{aligned}
& \infty \frac{1}{2} 0
\end{aligned}
$$$$
-k=0
$$

$$
\frac{3}{3}-1
$$




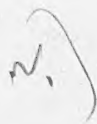

10

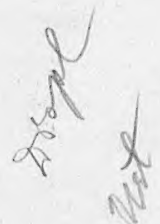

$\hat{s}^{1}$

Ni 


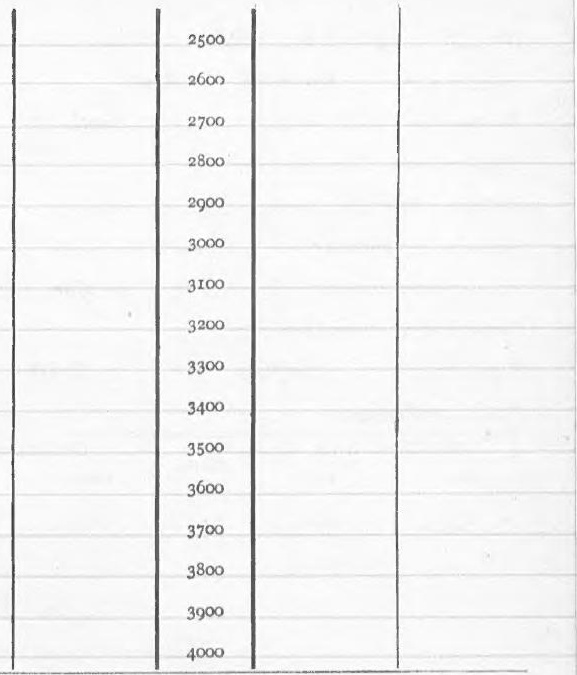

SERIAL TEMPERATURES.

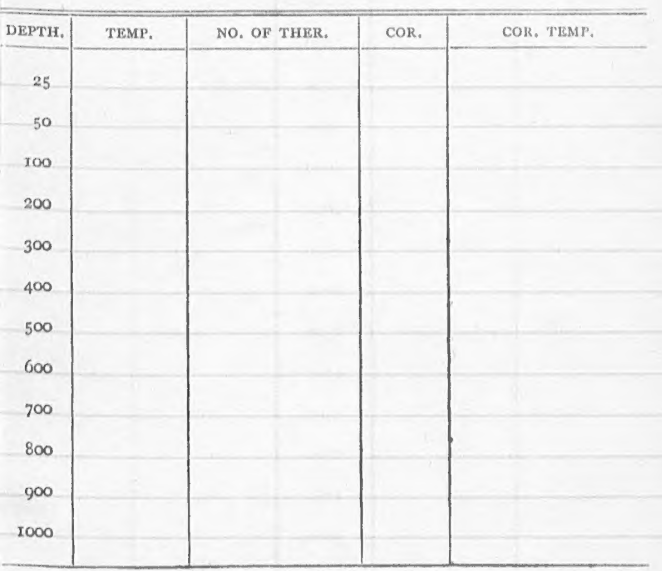

REMARKS : 


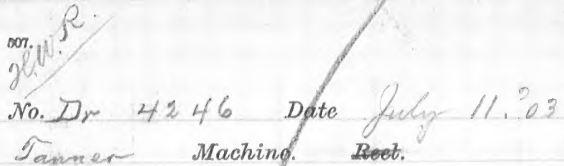

\section{Laminer Maching. Moet.}

Turns $118 \quad$ Cor. +5

Depth $/ 23$ Sin

shot or lead $26 \mathrm{Ms}$

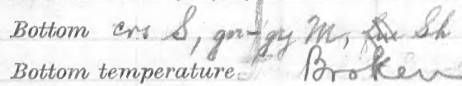

No. of thermometer 98239

Corrected temperature

Air $66^{\circ}$ Surface $62^{\circ}$ Drift 6/10"

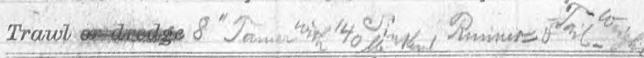

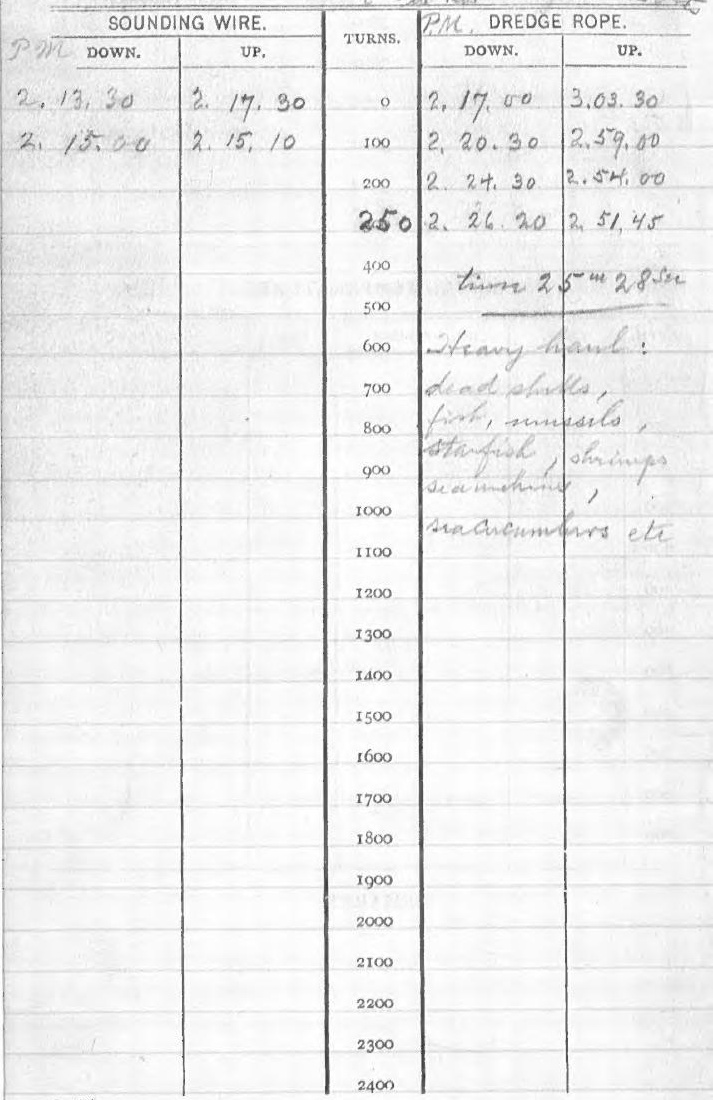


Kasaan Bay, alontea $3.0^{\prime}$

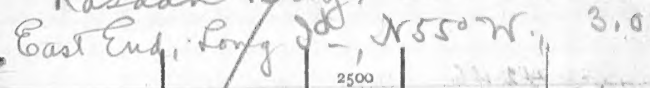

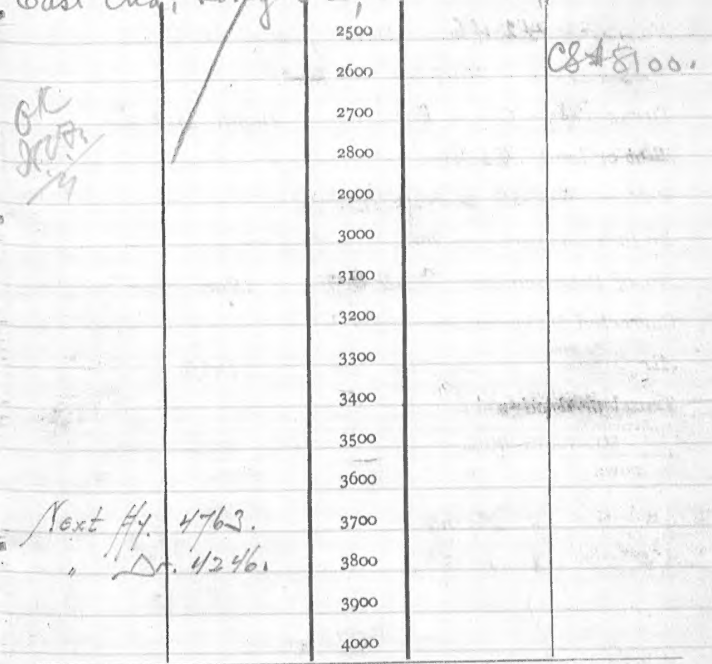

\section{- SERIAL TEMPERATURES,}

\begin{tabular}{r|r|r|r|r}
\hline \hline DEPTH. & TEMP. & NO. OF THER, & COR. & COR. TEMP. \\
\hline 25 & & & & \\
50 & & & & \\
100 & & & & \\
200 & & & & \\
300 & & & & \\
400 & & & & \\
500 & & & & \\
600 & & & & \\
700 & & & & \\
800 & & & & \\
900 & & & & \\
1000 & & & & \\
\hline
\end{tabular}

REMARKS: 
b07.

No.H, 4763

Fammor

Machine.

Date

Turns 97 Cor. +4

stobor lead 26 llas

Bottiom gn m, fine \&, brk Sh.

Bottom temperature 44,3

No. of thermometer 98237

Corrected temperature U४..1 ${ }^{\circ}$

Air $66^{\circ}$ Surface $62^{\circ}$

\section{Trow or aredge-}

P $7 /$ SOUNDING WIRE. DowN.

UP.

2. 53.00 2. $5 \% .00$

2. 54 , 20 2. $345^{\circ}$

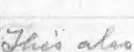

end of of

wartis

기. 4246

600

700

800

900

1000

I 100

1200

1300

$I A D O$

1500

1600

1700

I 800

1900

2000

2100

2200

2300

$240 n$
Cor. -0.2

Drift

Depth $101 \mathrm{fm}$ 
East End long os $820 \%, 1 \frac{9}{10^{\prime}}$

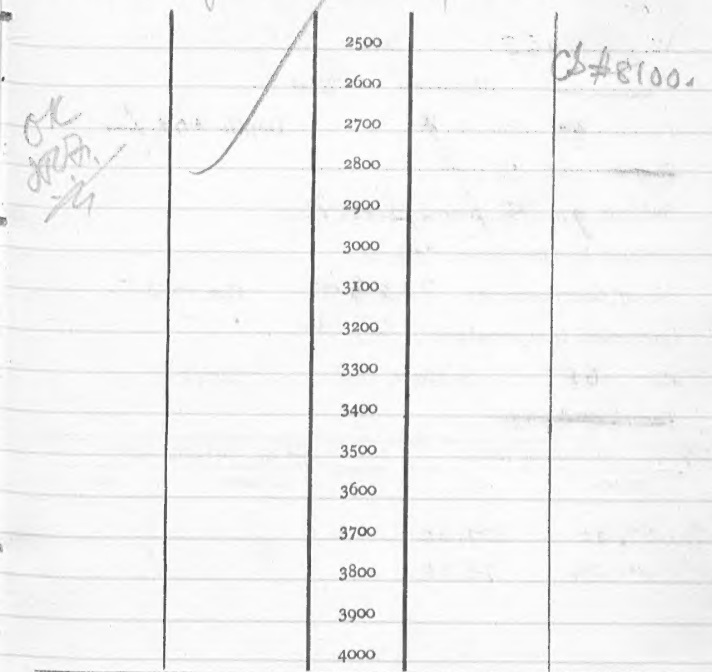

SERIAL TEMPERATURES.

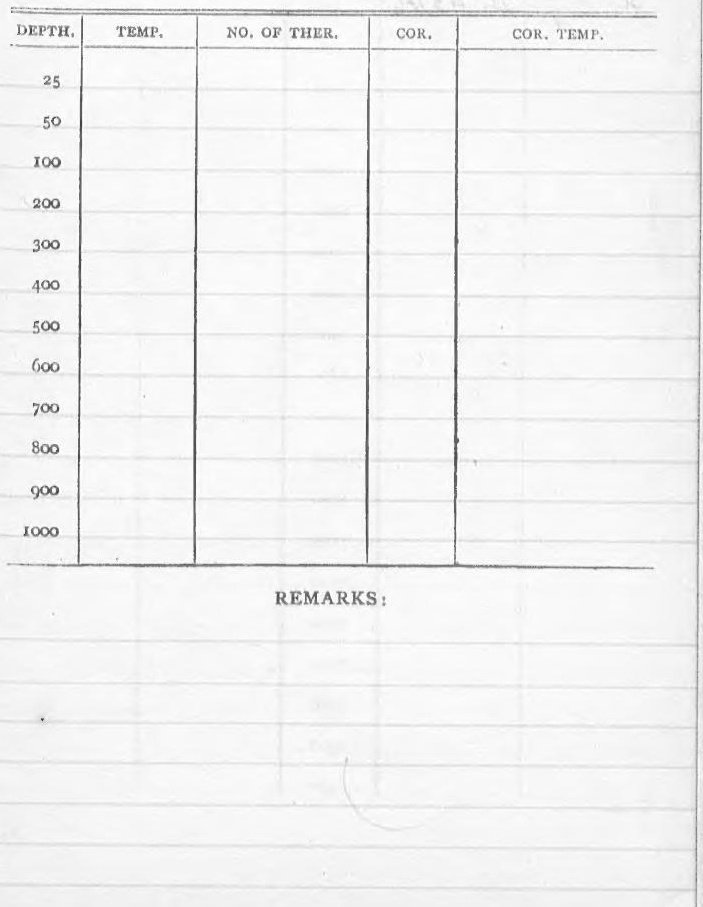


nort is

2

0.1

No. Trial

Tammar Machine. Reel.

Turns 42 Cor +2 Depth 44 fres

shot or lead

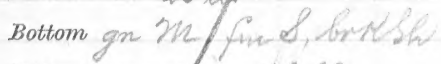

Bottom temperafure $46,6^{\circ}$

No. of thermometer $98 * 37 \quad$ Cor. -0.2

Corrected temperature 46,40

Air $65^{\circ}$ Surface $62^{\circ}$ Drift

Trawlendredge

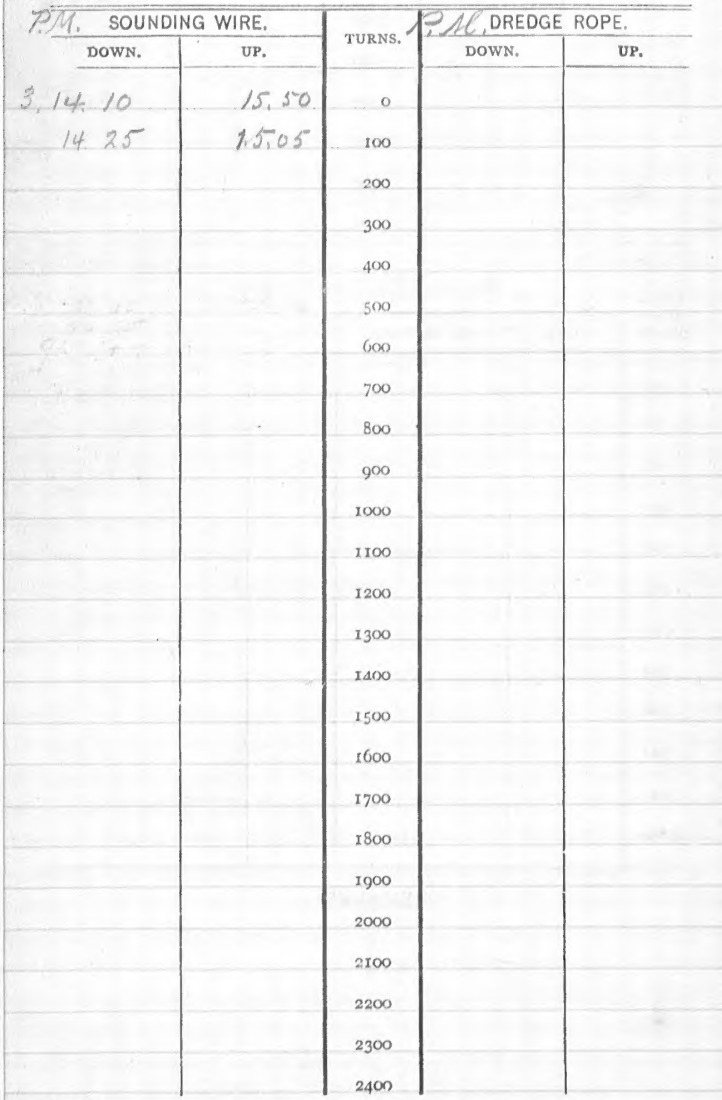




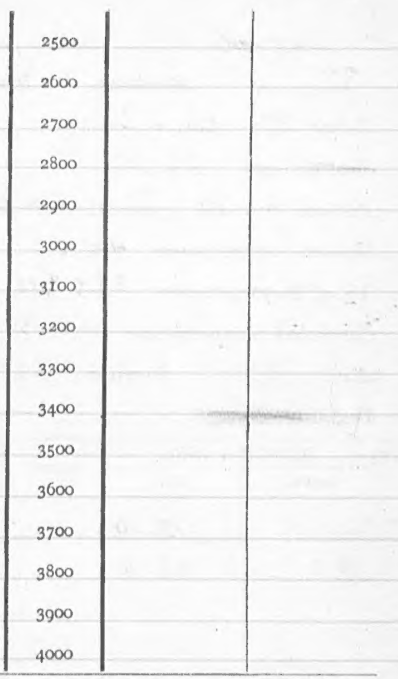

SERIAL TEMPERATURES

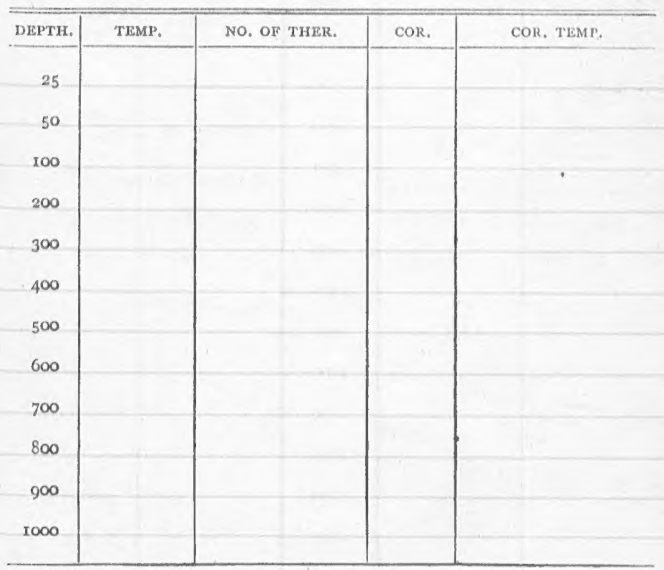

REMARKS : 
rot, 1 ?

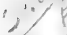

No. Dr

$$
\text { Tarn }
$$

$424 \%$

Date

Turns qI Cor. + it Machine.
$26 \mathrm{fb}$

Shotror lead

Bottom.

\#)

Bottom temperature

No. of thermometer

44,56

98237

Corrected temperature $\quad$ U $4,3$. Air..650 Surface $62^{\circ}$ Drift $4 / 10^{\circ}$

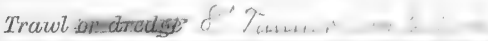

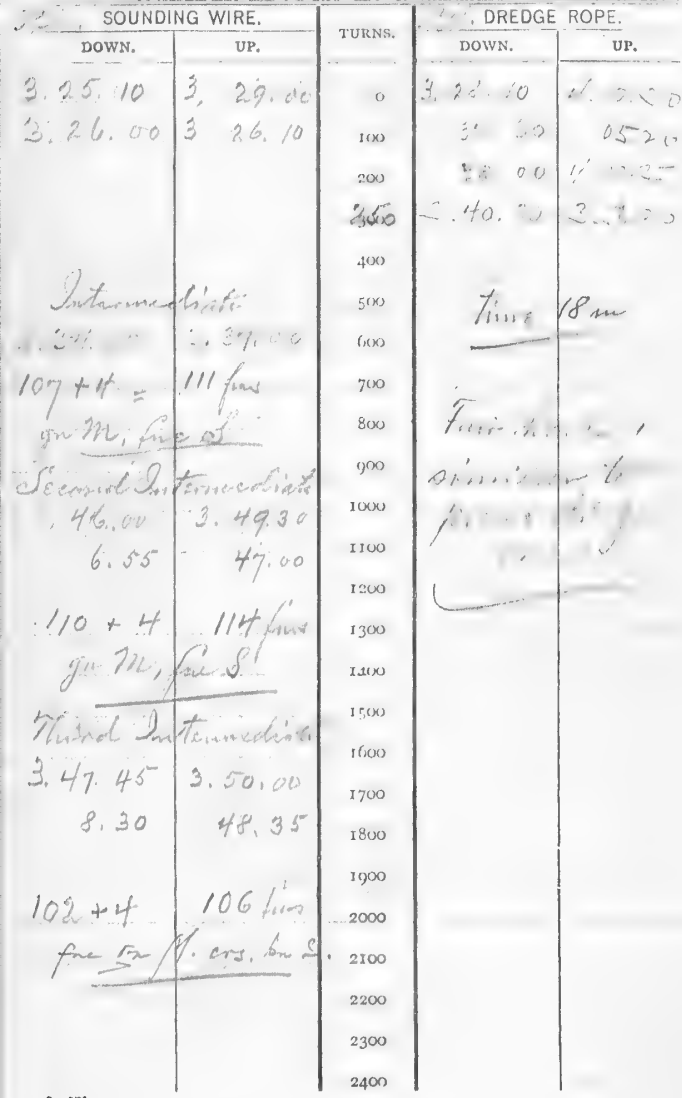

Cor. $-0,2$

(y) $20^{\circ}$ 


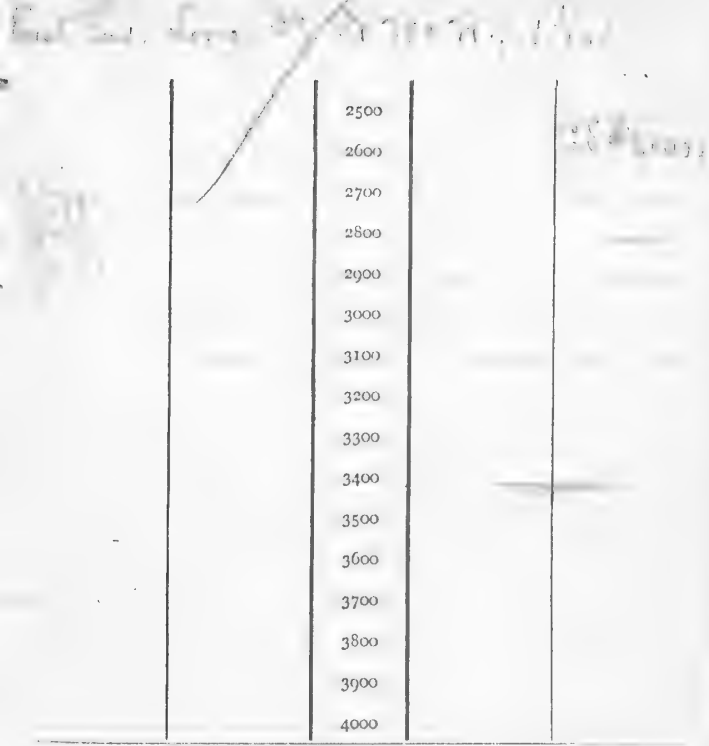

SEIIAL TEMPERATURES.

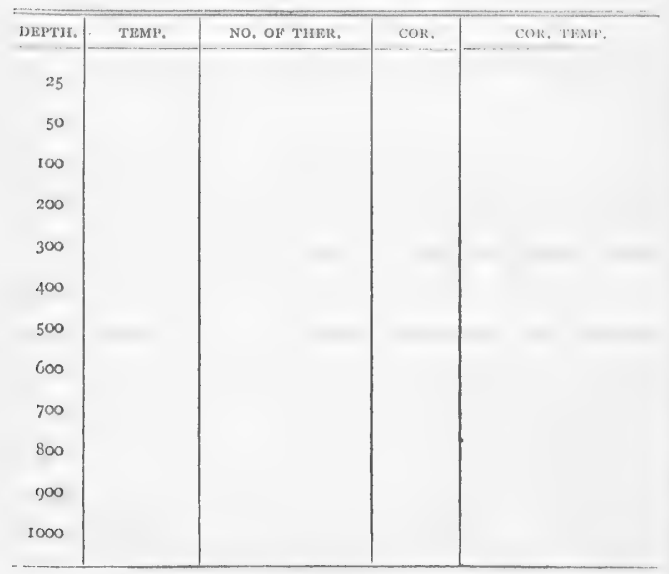

REMARKS : 
No.

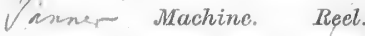

Turrus 86 Cor. +3

stwater lead $26 \%$

Bottom S. SK.

Bottom temperalure

\section{$\pi$}

No. of thermometer $9828 \%$. Cor.

Corrceted temperature

Air $65^{\circ}$ surface $63^{\circ}$ Drift

\section{Trawot-or-dredgo-}

$\frac{3 \text { SOUNDING WIRE. }}{\text { DoWN. }}$

$210,00+4$

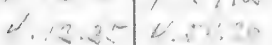

i

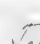

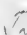

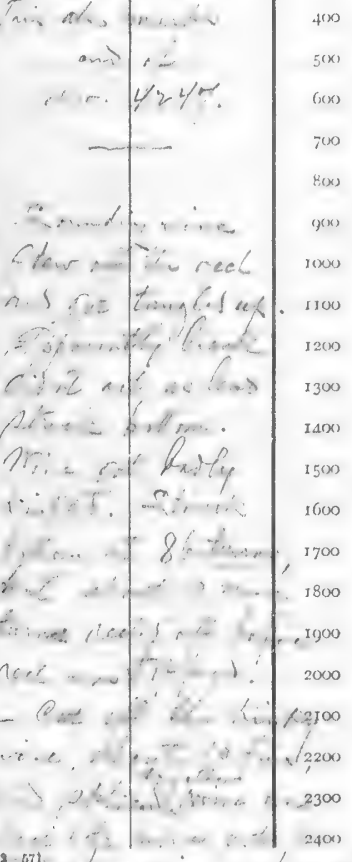

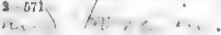




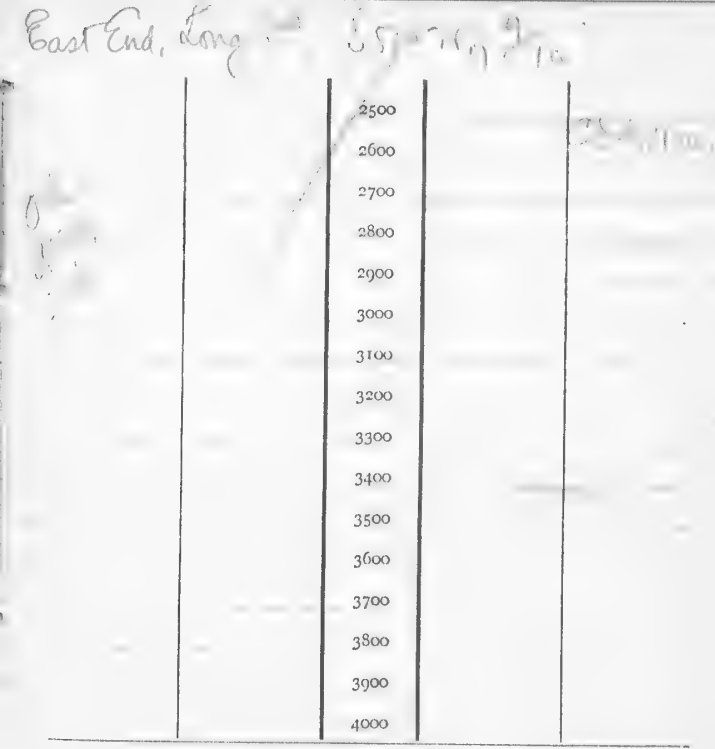

SERIAI, TEMPERATURES,

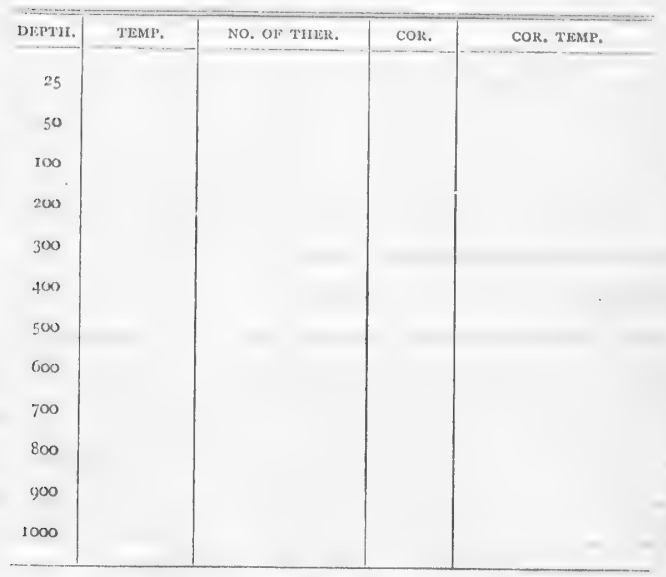

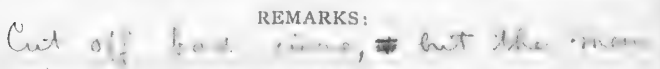

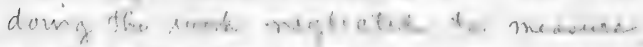

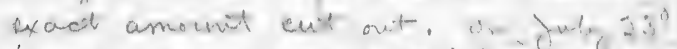

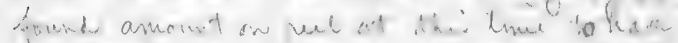

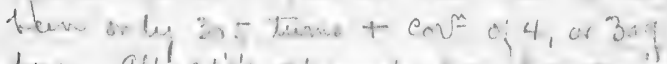

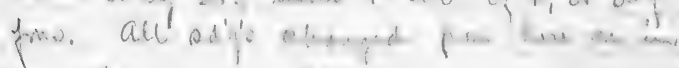
$\operatorname{sic}(*+, \pi)$ 
Sro. i7n 4248 Date Tammer Machine. Reel.

Throns 70 Cor. +1

shotor lead $26 \mathrm{flo}$

Bottom yiy $\geqslant 2$

Bottom temperature $43 \mathrm{3}$

No. of tharmometer

$9823 \%$

Corrceted temperature 42,86

$$
\text { Dopth } 71
$$

Air $56^{\circ}$

Surface

$55^{\circ} \cdot 0$

Cor. $-\mathrm{c}:$

Drift 1.0

Trrowl on dowelge.

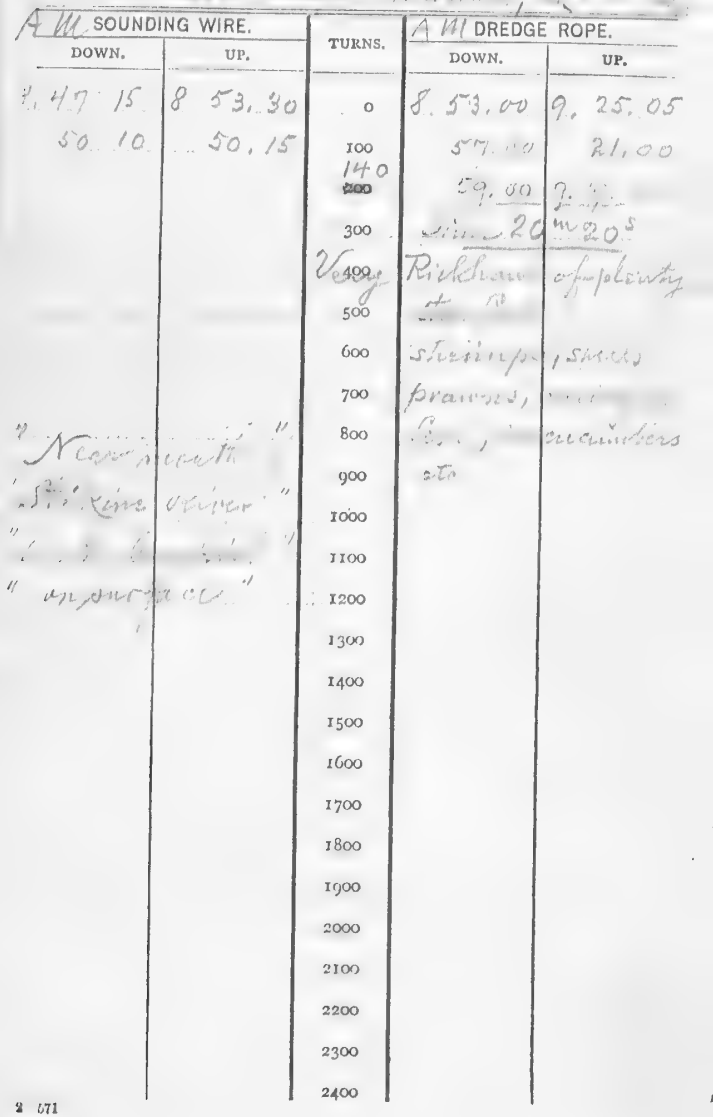


1.

No. $\div 76 \%$ Date

- ner Machine. Rél.

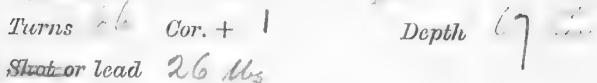

Botitom Q4. 72

Bottom temperature 43,50

No. of thermometer $9.83 .37 \quad$ Cor. $-\mathrm{a}_{2}$

corrected temperature $43,3 \%$.

Air 360 Surface. $55^{\circ}$ Drift

\section{Irawil or dwodrye}

A (i) SOUNDING WIRE.

$q_{1}$

Down.

$$
\begin{array}{ll|lll}
22 & 20 & 9 & 25, & 00 \\
2 & 10 & \approx & 3.15
\end{array}
$$
TURNS, DREDGE ROPE 
Non, :249 Dols Tamen Machine. Reel.

Thurns 78 Cor. +1 Depth 79 ?

staptor lead 26 C

Bottom gy $\% 2$

Bottom temperature $4 / 3, \&$

No of thermometer of ?

Corrceted temperature

Air : Surface is

Drift

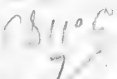

Trawl or diredse

1,1

7

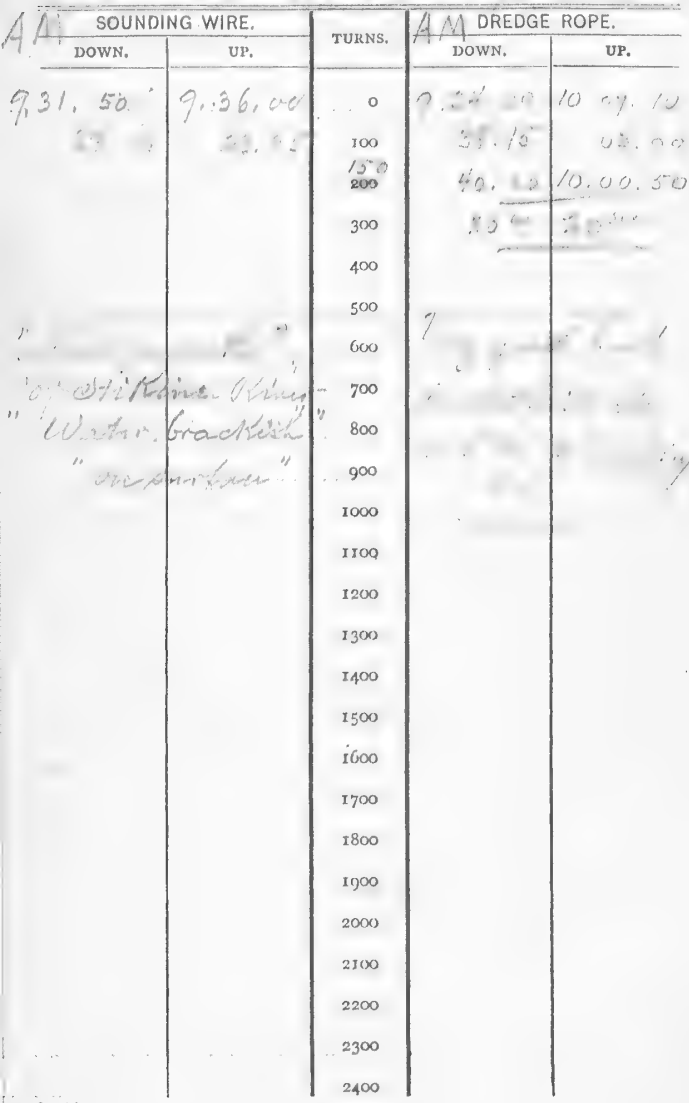




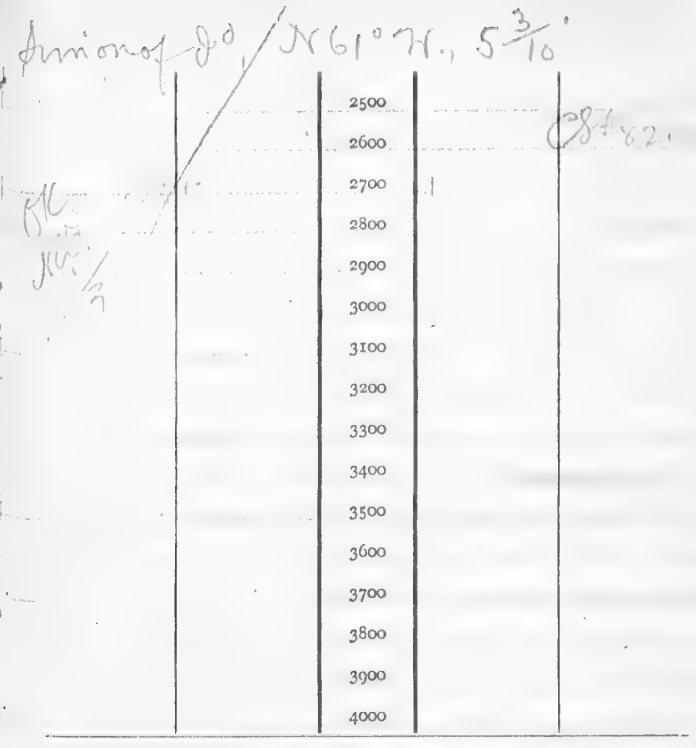

SERIAL TEMPERATURES.

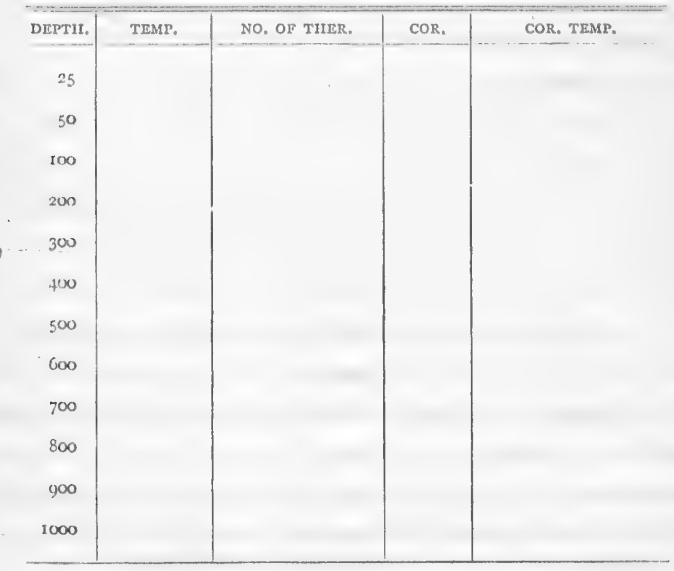

REMARKS : 
ro7.

0.

No.

Lft, $;$; Dale

Yan, Machine. I Reel.
Turns 69
Cor. +1
Shrot an lead
Depth 70

Bottiom $\quad$ g.y. 211

Bottom temperature 43,2 "

No. of thermometer 98 : $3 . \quad$ Cor. $\cdots$

Corrocted temperature

Air $56^{\circ}$ Surface $55^{\circ}$ Drift

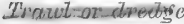

\begin{tabular}{|c|c|c|c|c|}
\hline \multicolumn{2}{|c|}{ SOUNDING WIRE. } & \multirow{2}{*}{ TURNS. } & \multicolumn{2}{|c|}{ DREDGE ROPE. } \\
\hline 111. nown. & $A$ Ur. & & DOWN. & ur. \\
\hline $\begin{array}{r}0.02 .50 \\
04.00 \\
4 \text { fin a so } \\
1 / 2 \\
\end{array}$ & $\begin{array}{l}10.07 .00 \\
10.04 .10 \\
90 \\
49 \ldots\end{array}$ & $\begin{array}{r}0 \\
100 \\
200 \\
300 \\
400 \\
500 \\
600 \\
700 \\
800 \\
900 \\
1000 \\
1100 \\
1200 \\
1300 \\
1400 \\
1500 \\
1600 \\
1700 \\
1800 \\
1900 \\
2000 \\
2100 \\
2200 \\
2300 \\
2400\end{array}$ & & \\
\hline
\end{tabular}


con.

No.Dr 4250 Date Samin Machine. Reel. Turns 6.5 Cor. 1 Depth . 66 int stwotor lead $26 \mathrm{Kt}$ Bottom giy ith

Bottom temperature

No. of thornometer. $9823 \%$

Corrected temperature. 42.8 .0

Air is 6 Surface is

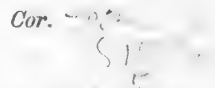

Trawl ondredse 8'/ 's

Drift

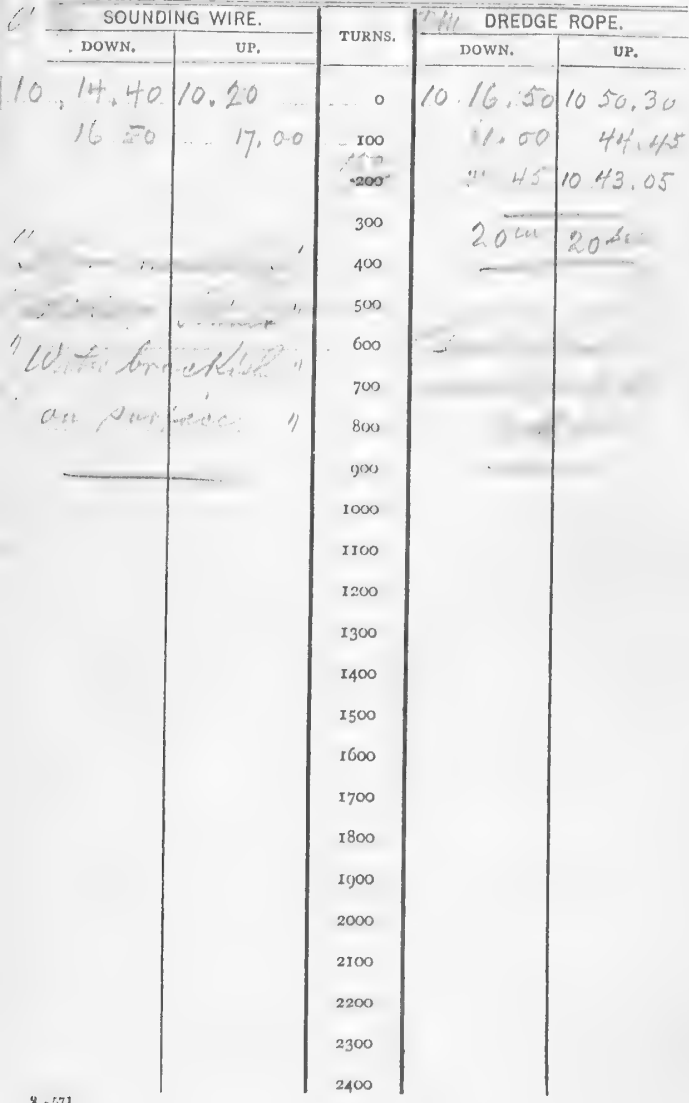




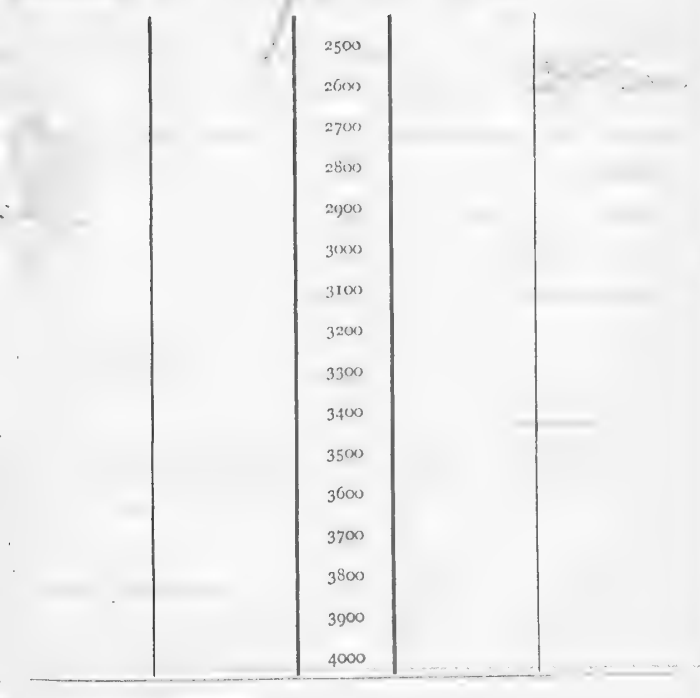

SERIAL TEMPERATURES.

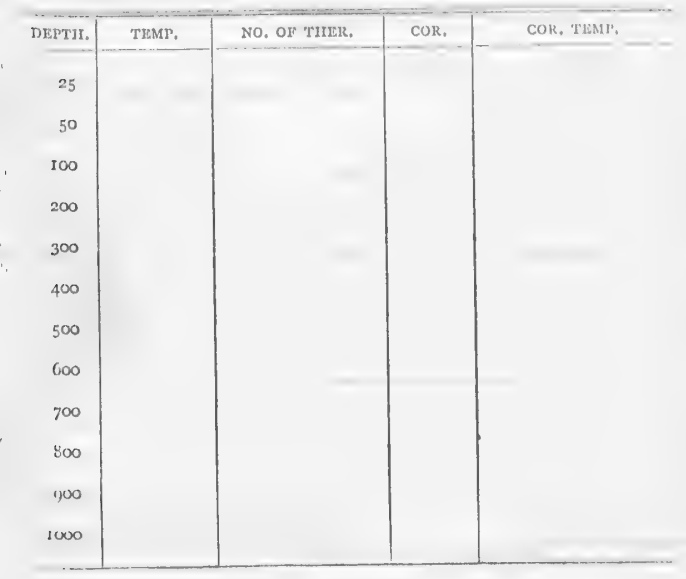

REMARKS : 
No.

a..

$\therefore \therefore$

Date

Machine.

Turns 60 Cor +1

Resl.

shoteor lead is 6 thes

Bottom gy. $1 / 26$

Bottom temperature 43,5

No. of thermometer 98297 . Cor. -0.2

Corrected temperature $43,3^{\circ}$

Air . 56० Surface...56 0 Drift

Trautear dredge

' SOUNDING WIRE.

DOWN.

UP.

$\because i \quad 10,11, \ldots$

$\therefore$. .

2. $4=0$

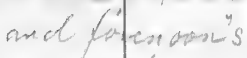

vel.

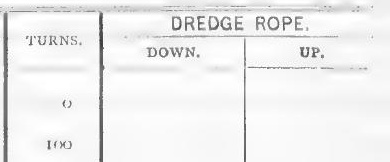

:00

$3(x)$

400

$5(10)$

$(x)$

700

$S(x)$

$y(x)$

1000

II00

1200

I 300

IAOO

I 500

1600

1700

I 800

I g)

2000

2100

2200

2300

$240 n$ 


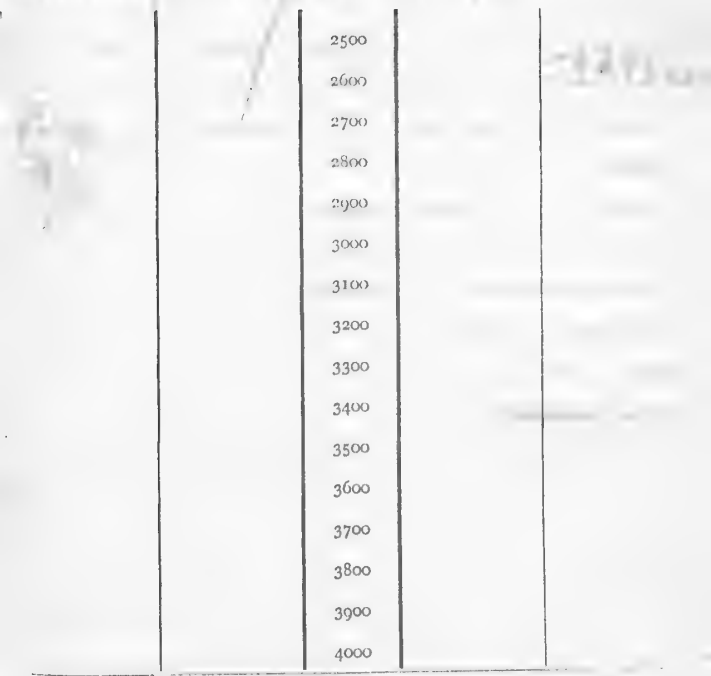

SERIAL TEMPERATURES,

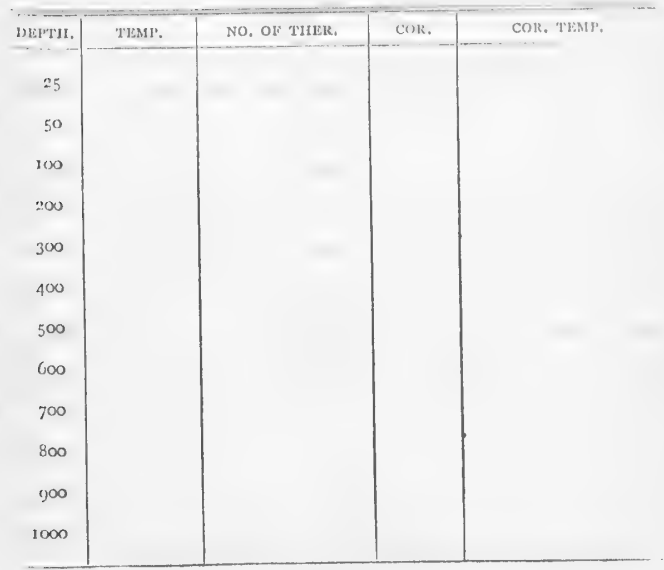

REMARKS: 
nort.

kil

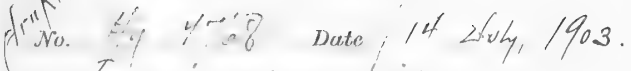

faminer Machine Reel.

Turns $\sqrt{8}$ Cor. $1 /$ Depth 59 frus.

Strotor lead 26 《"

Bottiom

Bottom temperature

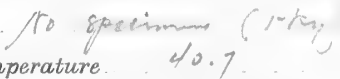

No. of therinometer

98,5

Corrected temperature

$$
98237 . \quad \text { Cor. A. : }
$$

$40.5=$
Air
Surface
$\sqrt{1}$
Drift

1frasul- of dredge

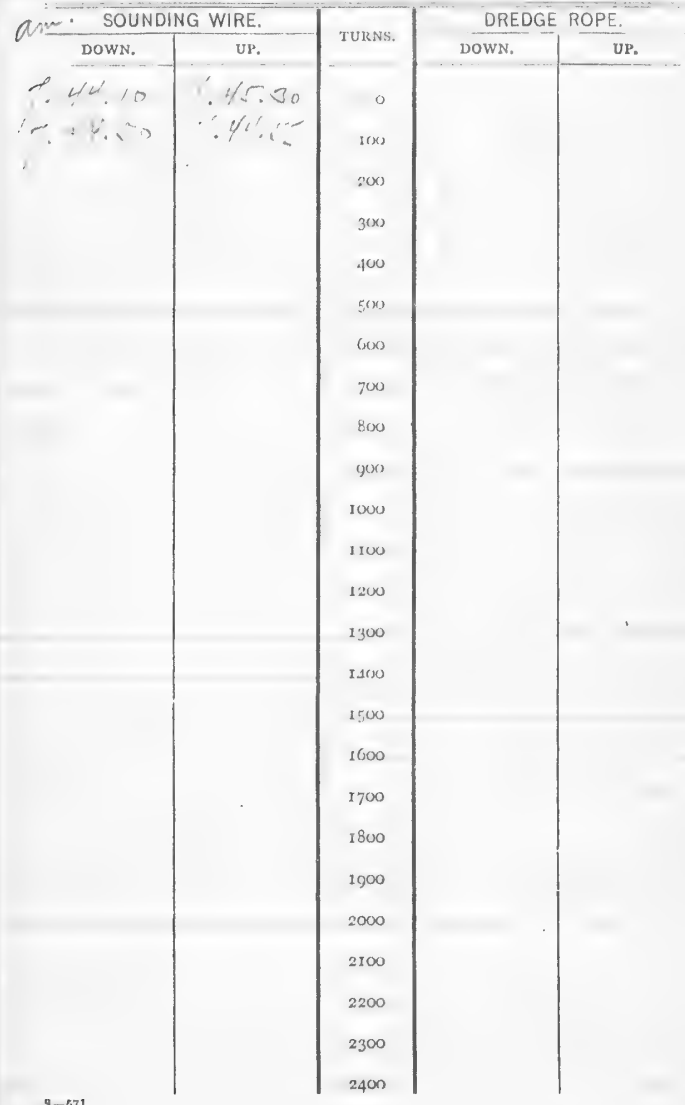


nor., $/$

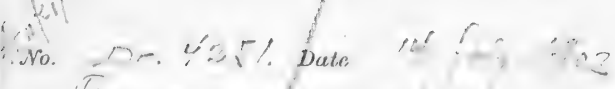
$(' A, \ldots$, Machine. Reel.

Turns $19-$ Cor $+: 3$ Depth $198 \ldots$.... Shot or lead $26 \%$

Bottom Nhey -

Bottom temperature

No. of thermometer

$96 ? 3 \div$ Cor.

Corrected temperature

Air $54^{\circ}$ Surface vrift 4

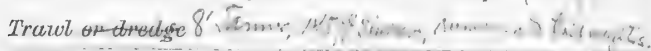

SOUNDING WIRE.

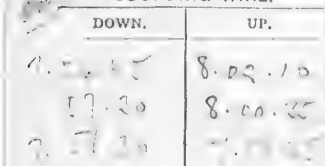

T. - i ?

2- 671

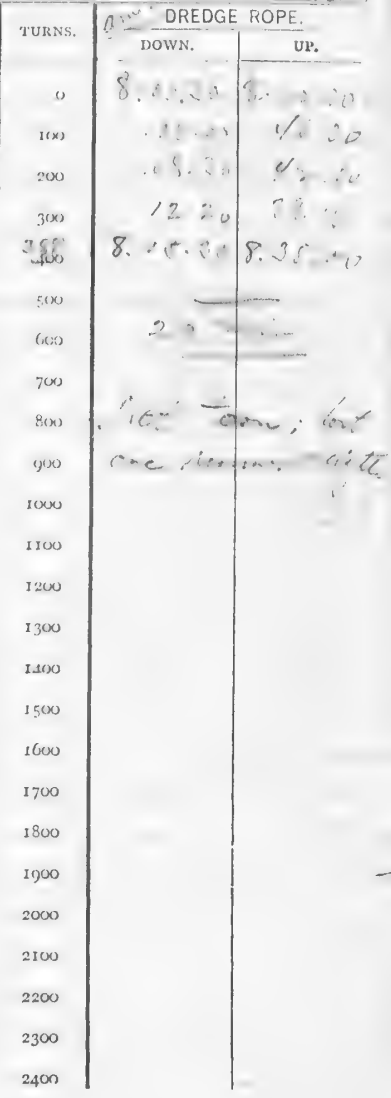




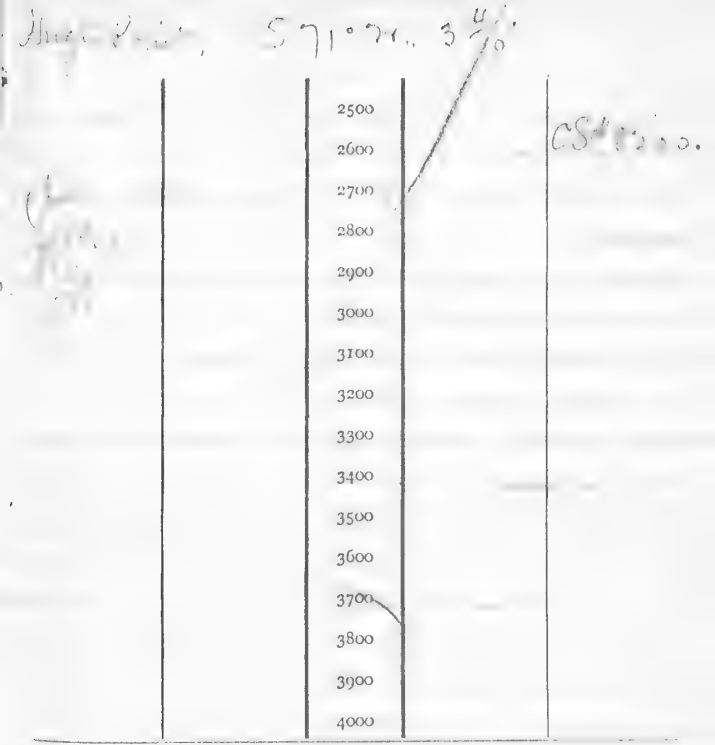

SERIAI, TEMPERATURES.

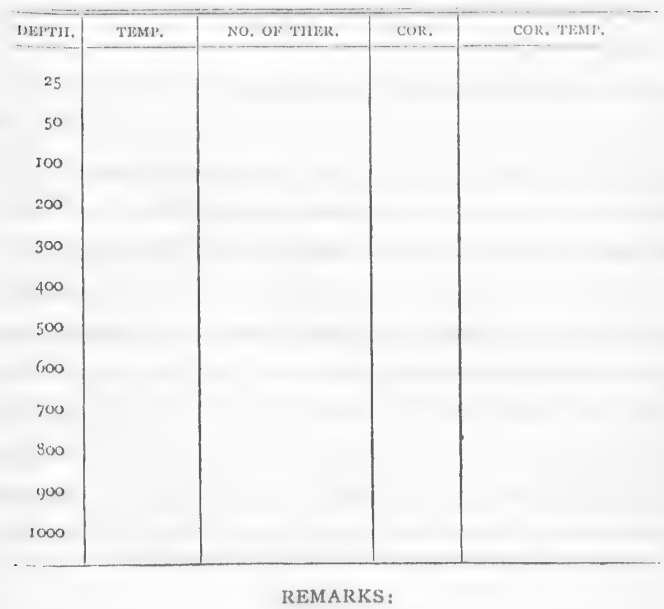


$n 07$.

so.

wo. Y/69 Date 1. Anfer Machino. Reel.

Turns
shot or lead 2.6

Bottom

Bottom temperature

$\begin{array}{ll}\text { No. of thermometer } & 98 \geqslant 37-\text { Cor. }-0.2 \\ \text { Corrected temperature } & 40.9^{\circ}\end{array}$

Air $\sqrt{40}$. Surface $\sqrt{\circ} \circ$ Drift

Frowot on-dredige

SOUNDING WIRE. DowN. UP.

5.47 .13

$4: 6$

40,20 (f) 7907 $i^{i} / 0-0$ $\because 6 i=2 i$

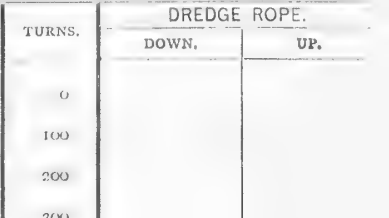




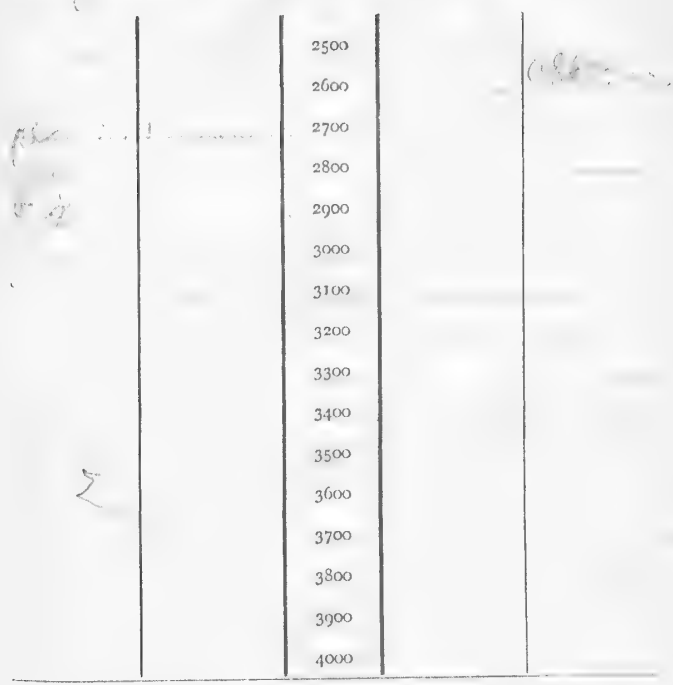

SERIAL TEMILRATURLS.

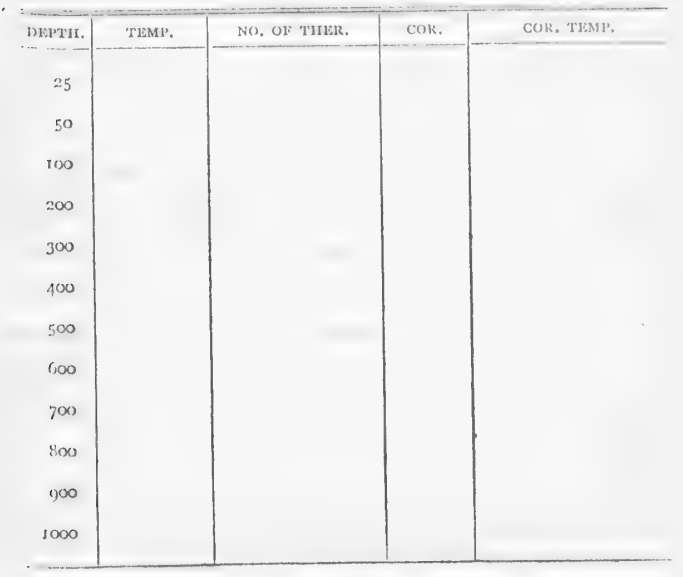

REMARKS : 
No.

$42: 2-$ Dat

14 lin, 03.

I Ane $r$ Machine. Reel.

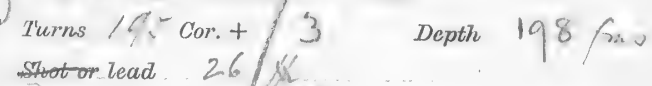

\section{Bottom}

Bottom temperature

So. of thermoineter.

Corrected temperature

Air is Surfaco

Trawl or dredge 8,1 Amme

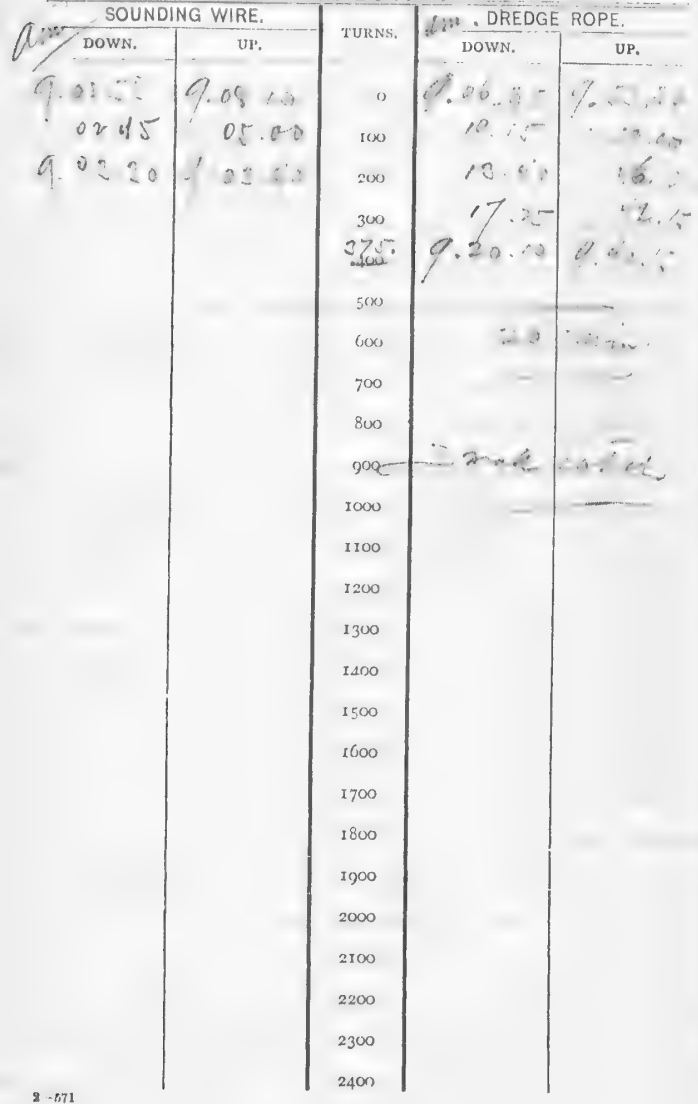




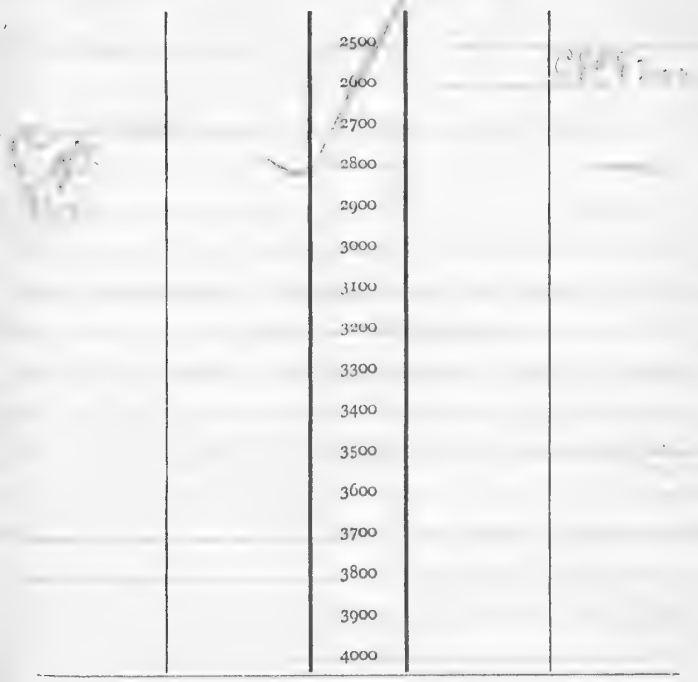

SERIAL TEMPERATURES.

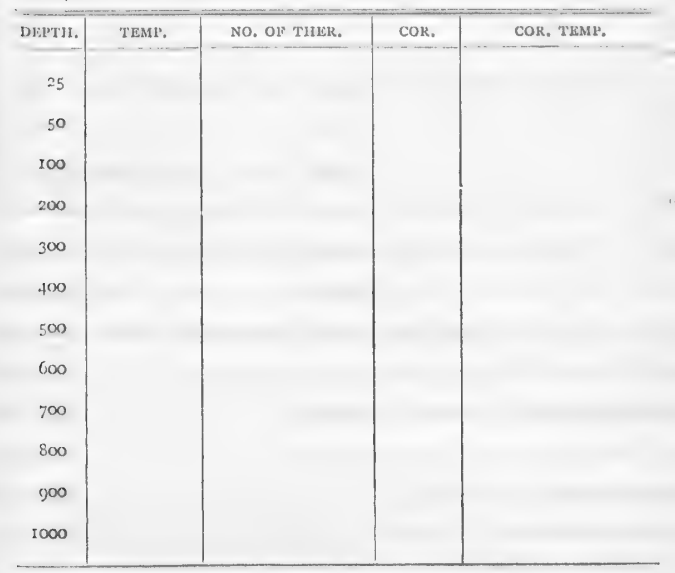

REMARKS : 
son.

be

No.

\%. Date

Finsfur Machine / Reet.

Turns 1.15 Cor. 3 Depth 201 in.

Storad 26

Bottom

Bottom temperature

$$
40.1
$$

No. of thermometer

Corrected temperature

Air Sf Surface $<$ Drift

Trawl or dredge

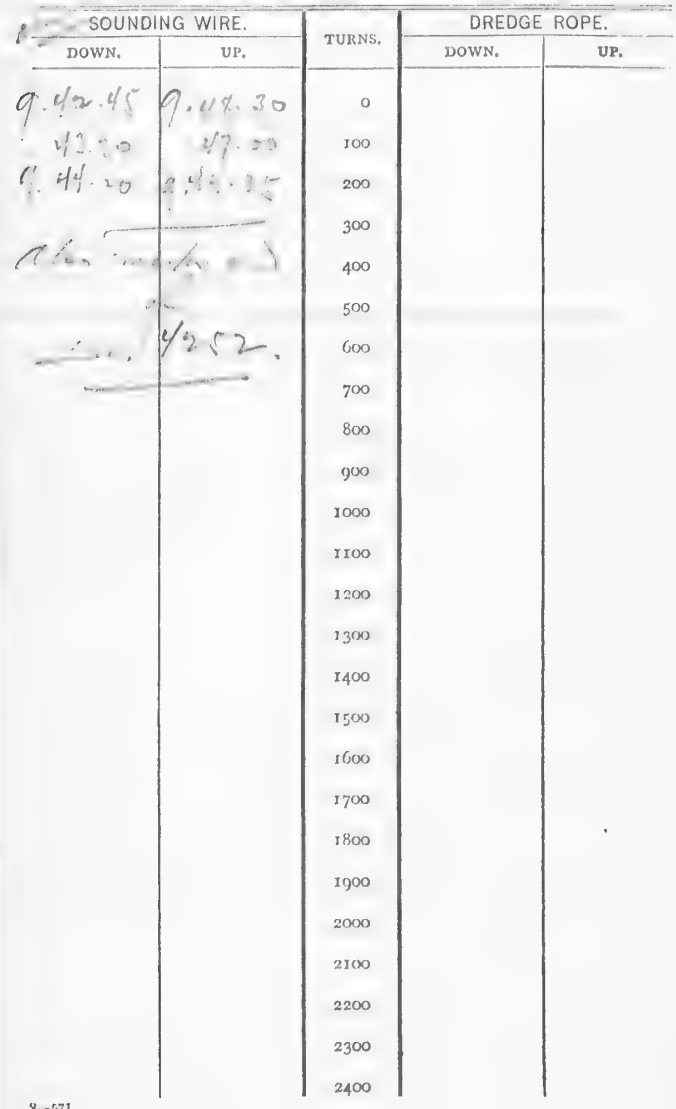

- 123 Cor. $-1 .=$

$3+10$ 


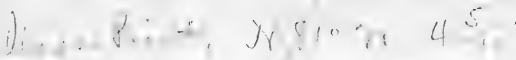

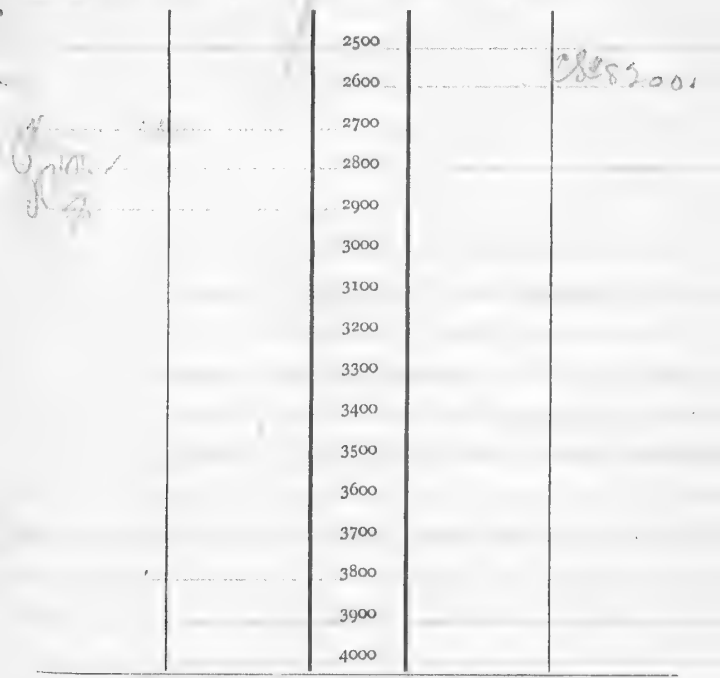

SERIAL TEMPERATURES.

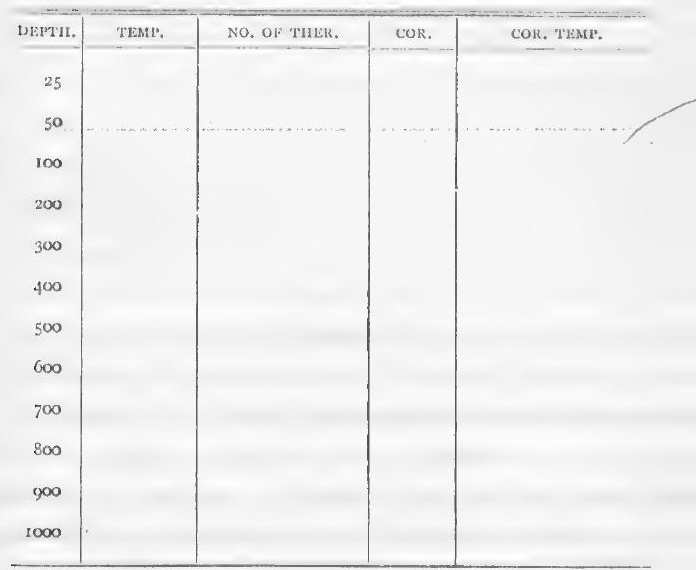

REMARKS : 
No. I, ' Date

$16 !, \quad 16: ?$

¿ Mimer Machine Reel.

Thros

$\mathrm{Cor}+\mathrm{?}$

Dopth / 68 fm.

कton or lead 26 \&!

Botitom

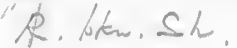

Bottom temperature

No. of thormorneter 41.

Corrected temperature

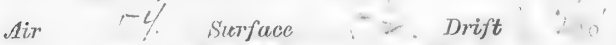

Trawt or dredge

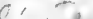
$4: 5 \div$. Cor. $\therefore$ SOUNDING WIRE. DOWN. U1. TURNS. 17.<smiles>c1ccccc1</smiles>

$\sqrt{1.10}$

$10.51 .3010 \ldots 1.21$ II:W DREDGE ROPE. \begin{tabular}{|l|l|l|}
\hline DOWN. & UP. \\
\hline
\end{tabular}

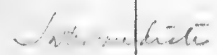

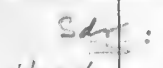

$$
\text { , }
$$

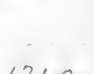

200

300

$13.35 \times$

$$
\text { (n) }
$$

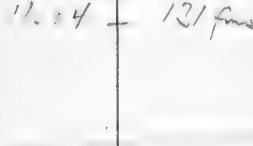

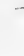

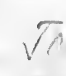

-

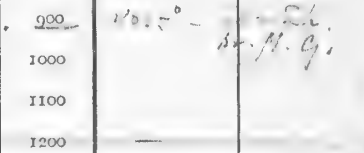




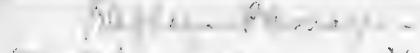
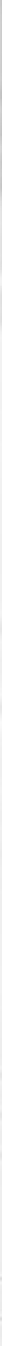

REMARKS : 
No.

177, Date

Tann: Machine reel.

Turns

Cor. +2

Depth $/ 36$

stert on lead

Bottom

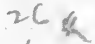

Bottom temperature

s.tin sc.

$$
\text { 4o. } 7
$$

No. of thermometer

$$
\because 1 \div 3
$$

Correctod temperalure

$$
\text { c. } 0.50
$$

Air

Sterface

Drift

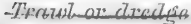

$\therefore$ SOUNDING WIRE $\because$ HOWN.

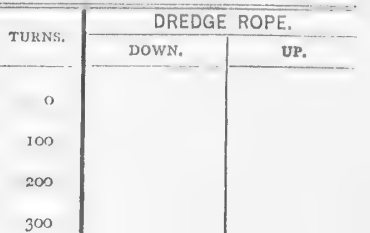
DOWN. UP.

UP.
$+5$
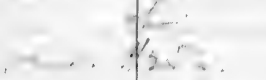


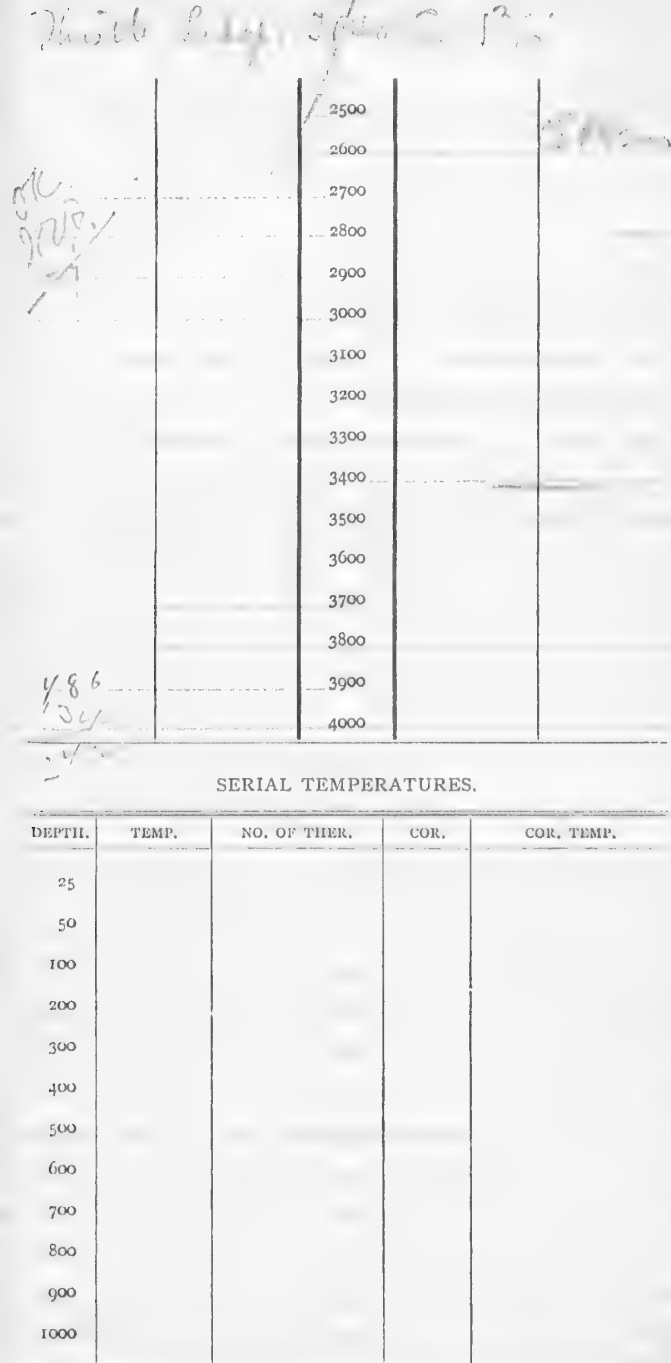

REMARKS: 
No. i) 2 : 2 Date

J'. Muctuine. Reel.

Thurrs 44 Cor. +1

ghoteor lead $261 \mathrm{ll}$

Depth 4t5 Hico

Bottom gn 72

Bottom temperature.

40,2

No, of therimoncter.

Corrected temperature

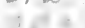

$40^{\circ}$

Cor. $-\mathrm{C}, 2$

$(5 \xi$ $(5 \xi)$

Drift

Air. Surfuce

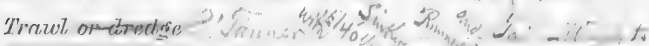
SOUNDING WIRE.

DOWN.

$\therefore 19-10$

is:

UP.

$0,-45$

$13: \div$

\begin{tabular}{|c|c|c|}
\hline \multirow{2}{*}{ TURNS. } & \multicolumn{2}{|c|}{$l$ DREDGE ROPE. } \\
\hline & DOWN. & UP. \\
\hline & $101, \ldots$ & $\because \quad-\quad y$ \\
\hline
\end{tabular}

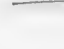




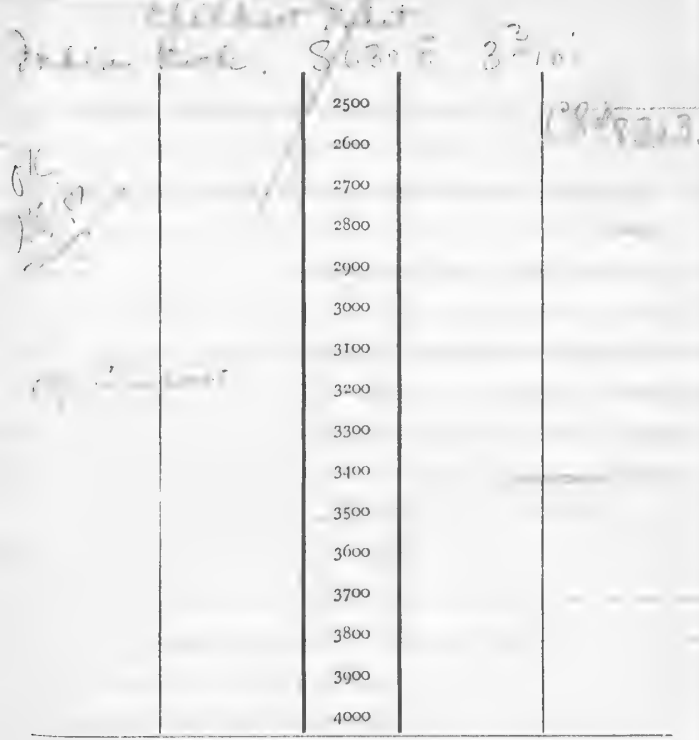

SERIAL TEMPERATURES,

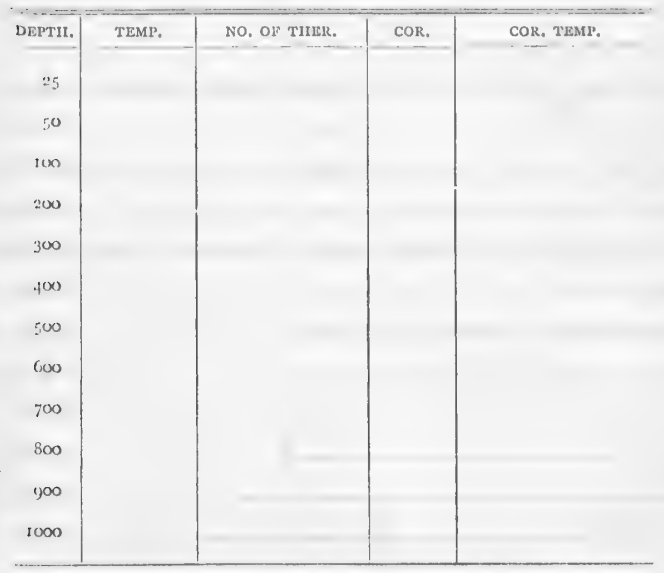

REMARKS : 


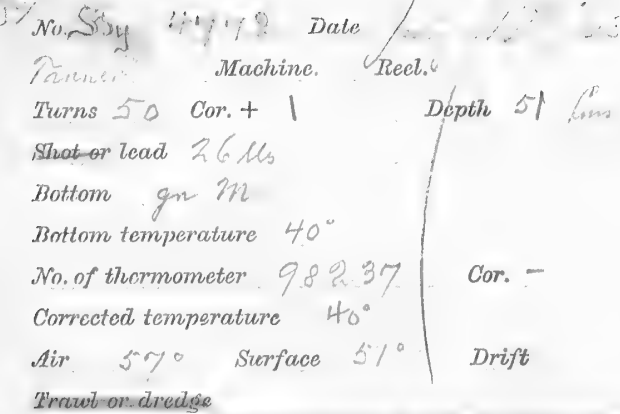

Cor. -

Drift

DOWN. UP.

$10,38,3$. 39.10

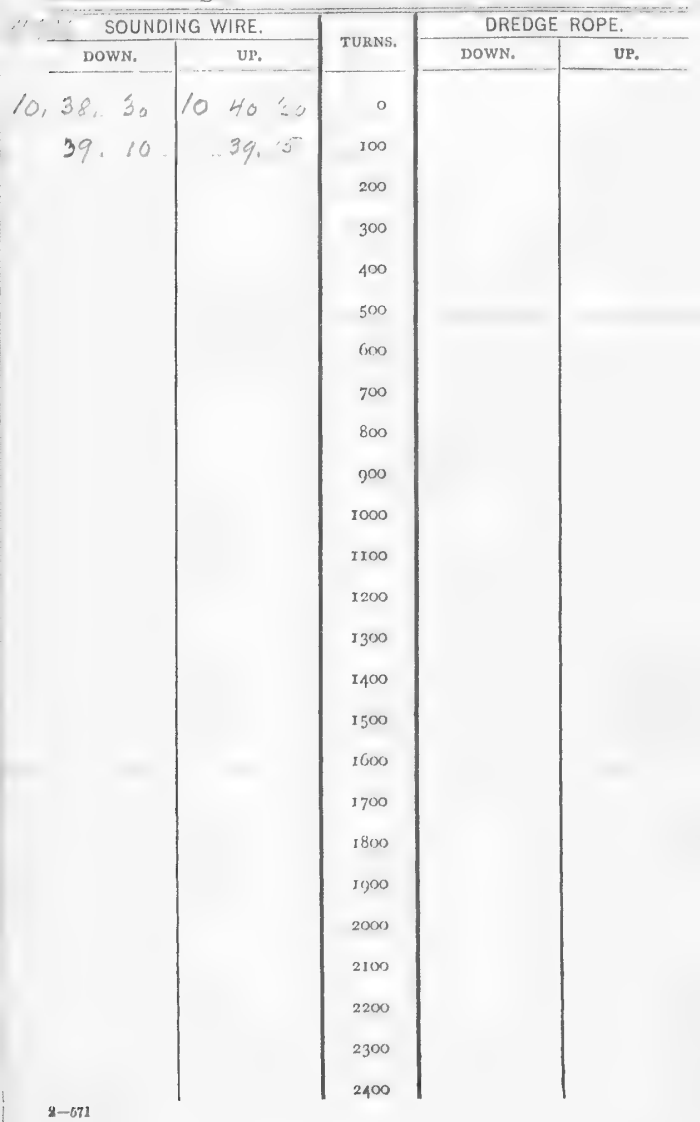




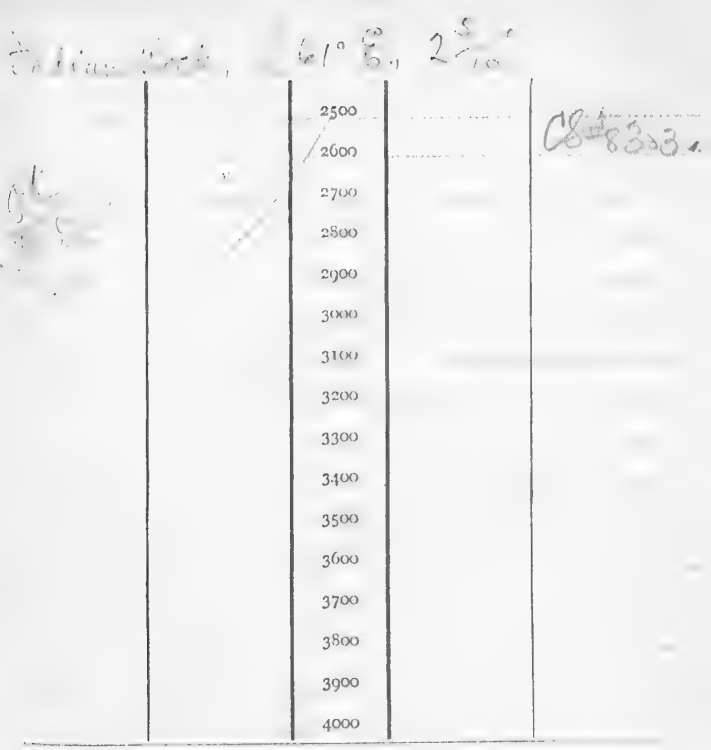

SERIAI, TEMPERATURES.

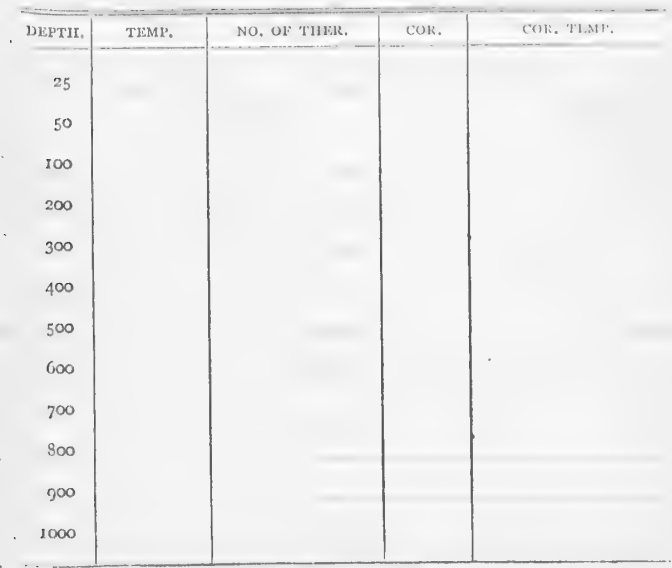

REMARKS : 

Daina Dult

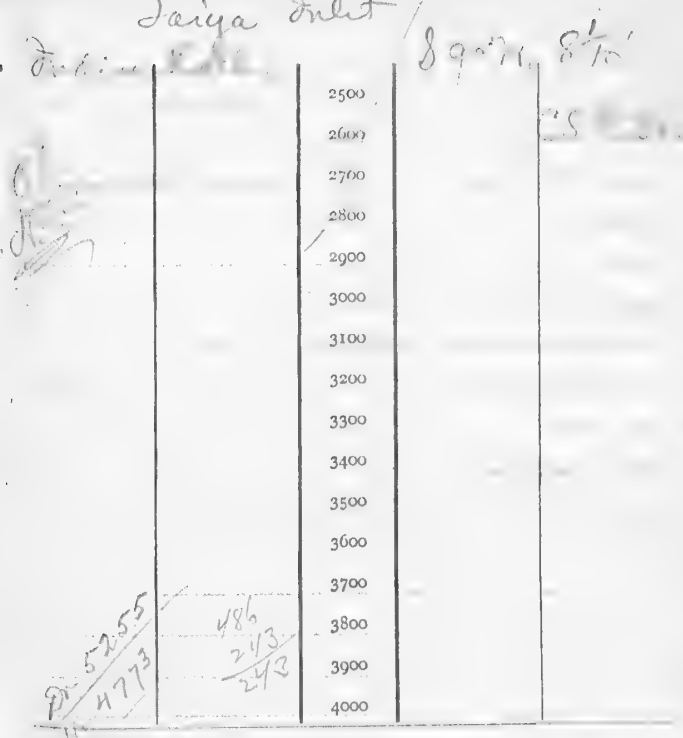

SERIAL TEMPERATURES,

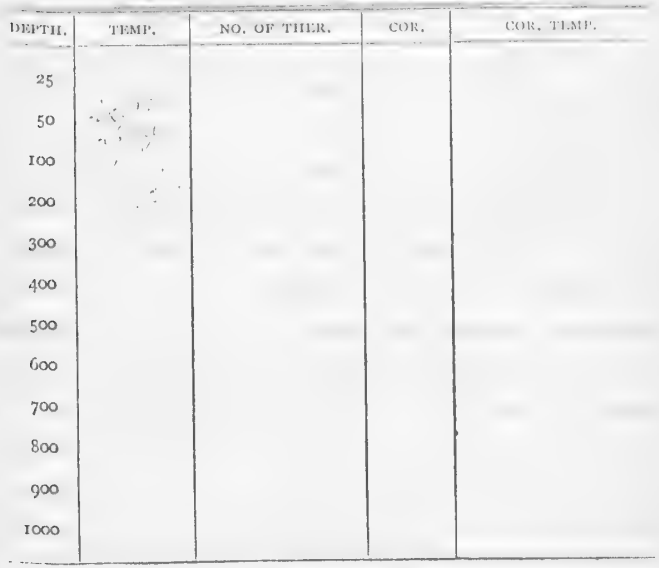

REMARKS: 
No.

$$
\therefore \text {. Date }
$$

$\checkmark$ mmer Machine Reel

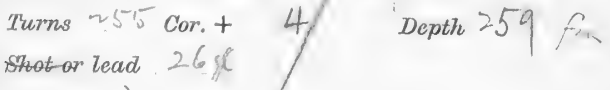

Bottom «)

Bottom temperature

No. of thermometer

Corrected temperature

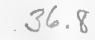

$98 \geq 3 \%$

Cor. -

$36.8^{\circ}$

Air . $60^{\circ}$ Surface $\checkmark \mathrm{V}^{\circ}$ Drift

\section{Trawt-or diredse}

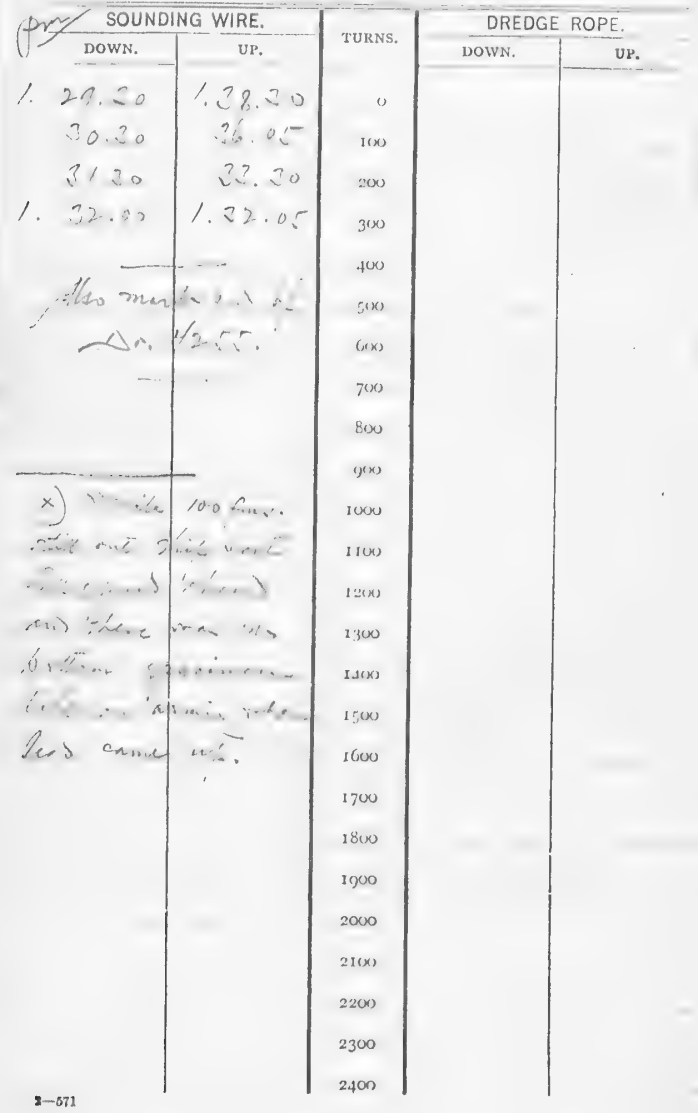


No.

- Tammer Machinc. Reel.
Turn
11
Cor. +1 .
Depth 72 fins

Shatar lead $26 \%$

Bottom $5 \mathrm{M}$.

Bottom temperature

38.3

No. of thermometer

98237 Cor. -

Corrected temperature

38.3

Air $62^{\circ}$ Sturface $9^{\circ}$ Drift

Trowul ar dredge Ino SOUNDING WIRE. DOWN. UP.

\begin{tabular}{c|c|c}
\hline TURNS. & DREDGE ROPE. \\
\hline & DOWN. & UP. \\
\hline & & \\
\hline
\end{tabular}

$2.6 ?-2.97 .10$

$2 \cdot 5^{+} .10 \quad 2.57 .12$

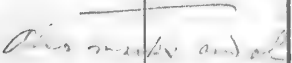

200

$1 / 256$

,

$3(x)$

4)

500

(100)

700

800

goo

1000

1100

1200

1300

IA(x)

1500

1600

1700

I 800

Igoo

2000

2100

2200

2300

$240 n$ 


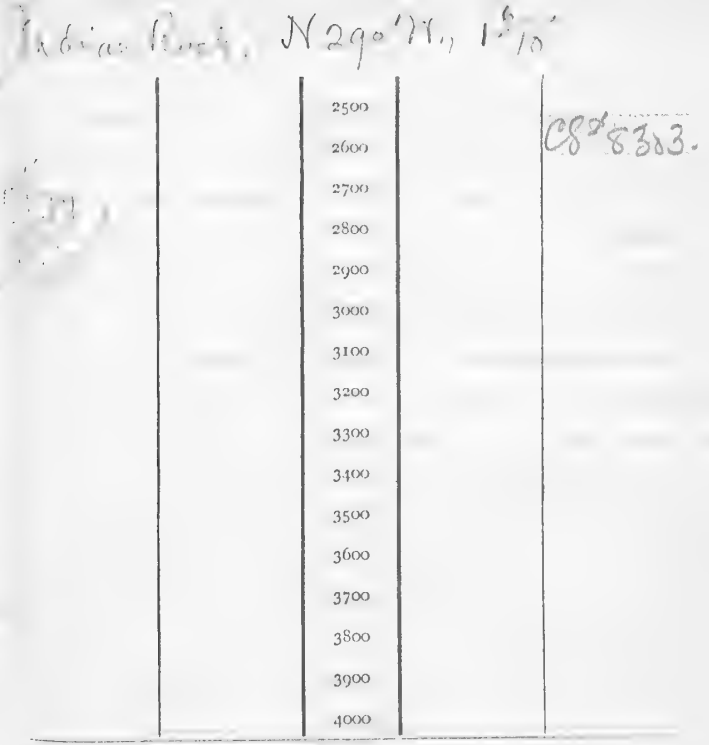

SERIAL, TEMIPIEATURES.

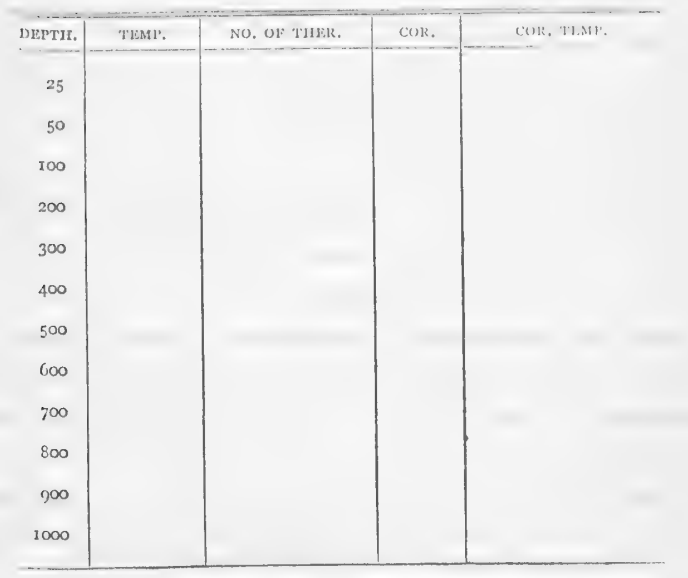

REMARKS : 

507

No. Dr Date 5 Rech.

Turns

Cor. +

1. In-1 Depth Sono fros

S7rot or lead

Bottom frow el axt

meed

Bottom temperalure

$\mathcal{N}$ o. of therrnometer

$\{r$, thenencor.

Corrected temperature

Air

Sierface

Drift

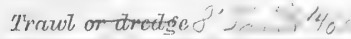

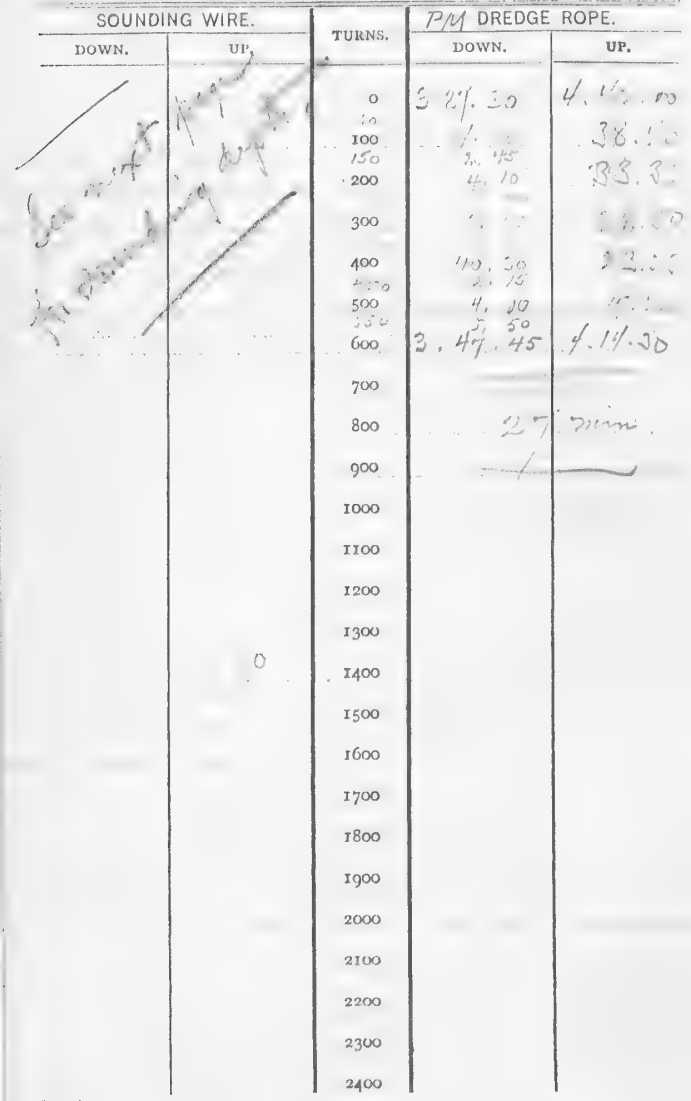


ClearPoint, $\mathcal{N}_{16} \cdot \varepsilon, 3^{5} 0^{\circ}$

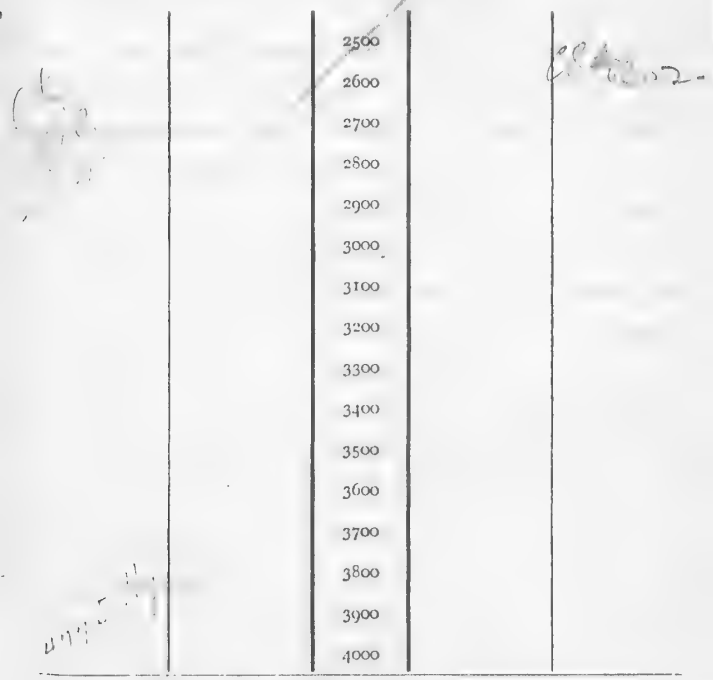

SERIAL TEMIERATURES.

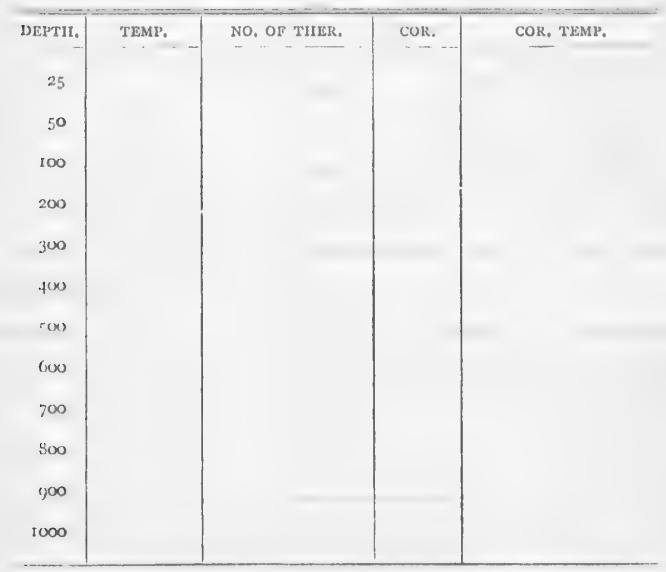

REMARKS : 


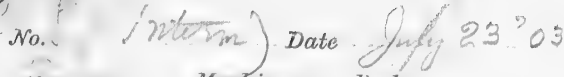
Machine. Reel.

Turns 20 Cor + Depth 300 jim

Shator lead

Botiom

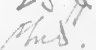

Bottom temperalure

No. of thormometer

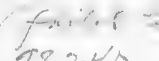

Corrected temperature
Air . 63.
Sierface
$\sqrt{3}$ Drift

trawt or diedge

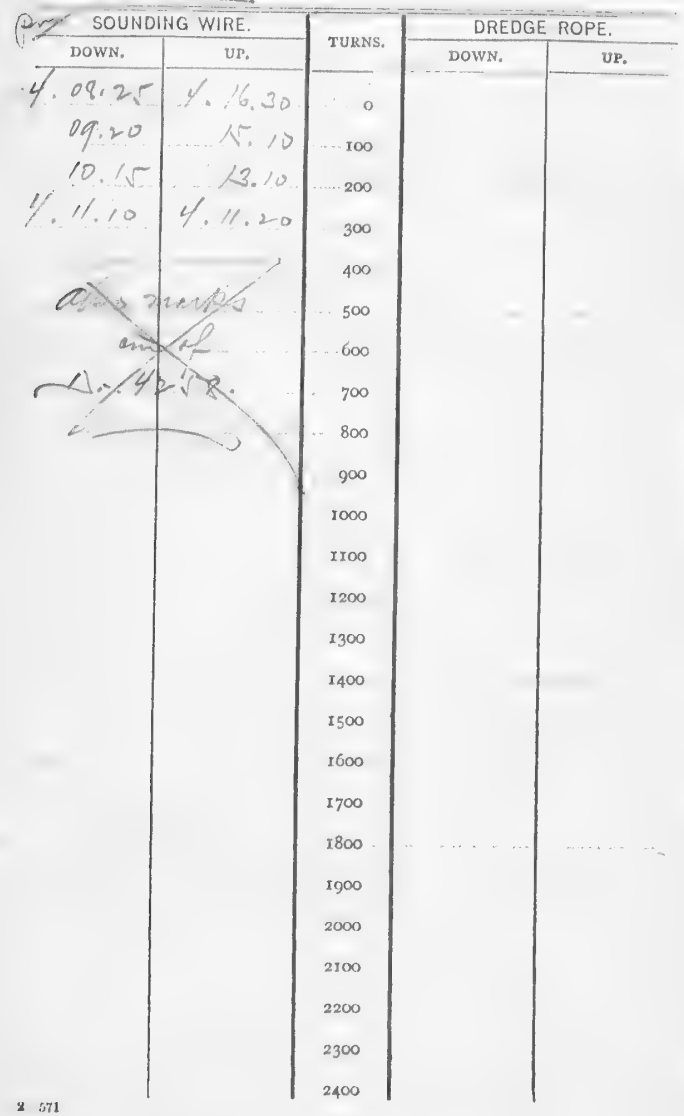


Jumer' Machine. Recl.

Therns 3 of Cor. 7 Depth 313

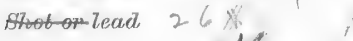

Bottom

Bottom temperature

Non. of thermometer.

Corrected temperature

Sir 63 Sterface Trift

Aretut or dretbo-

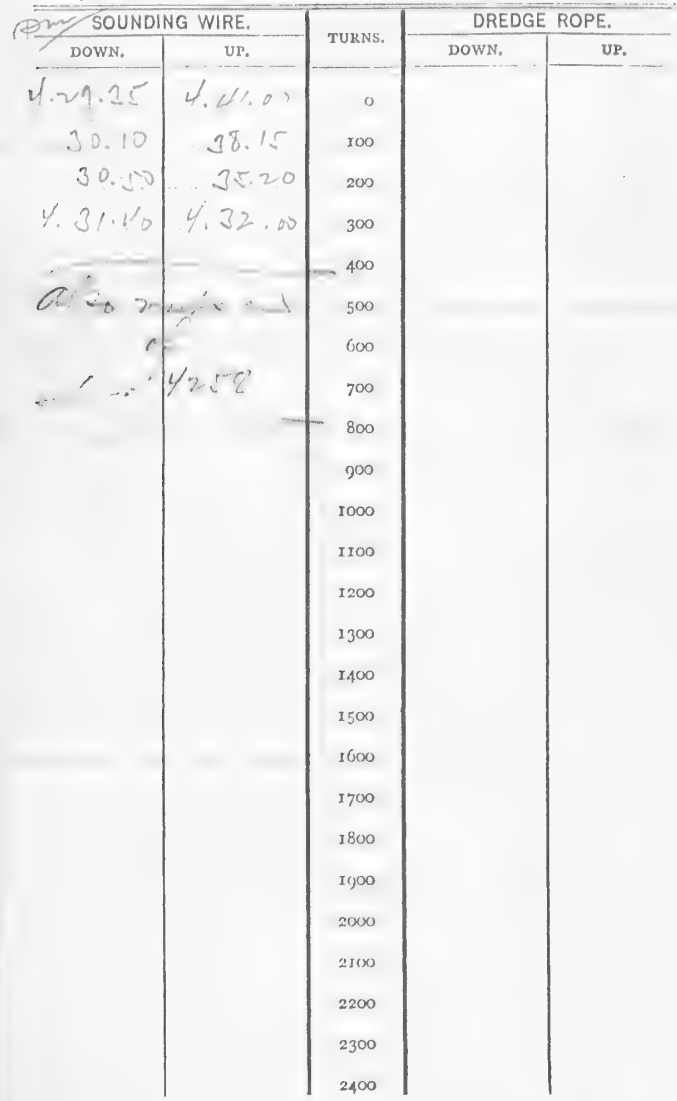


fion.

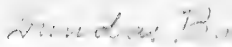

No. Li" $=q$ Dr Date

\% Muctivine. Recl.

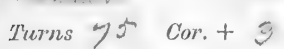

stombor lead 24 les"

Botitom buit of, $P$, of.

Bottom temperature 4fit. 5;

No. of thosmometer o 3 i :

Corrected temperalure. $4 L F.]^{\circ}$

Air Ke Surface $50^{\circ}$

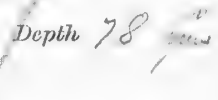

Trawt or dredge.

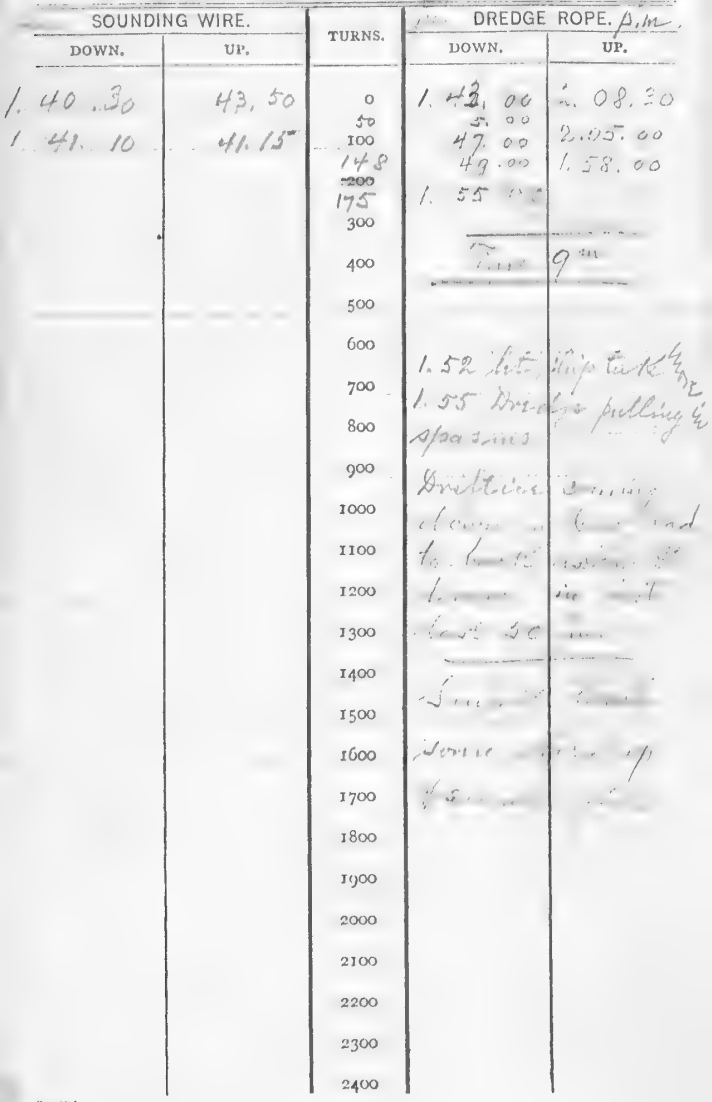

Cor. $\stackrel{-1}{1}, 2$.

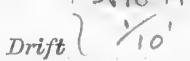


Dundar Bry - Oeg Horis

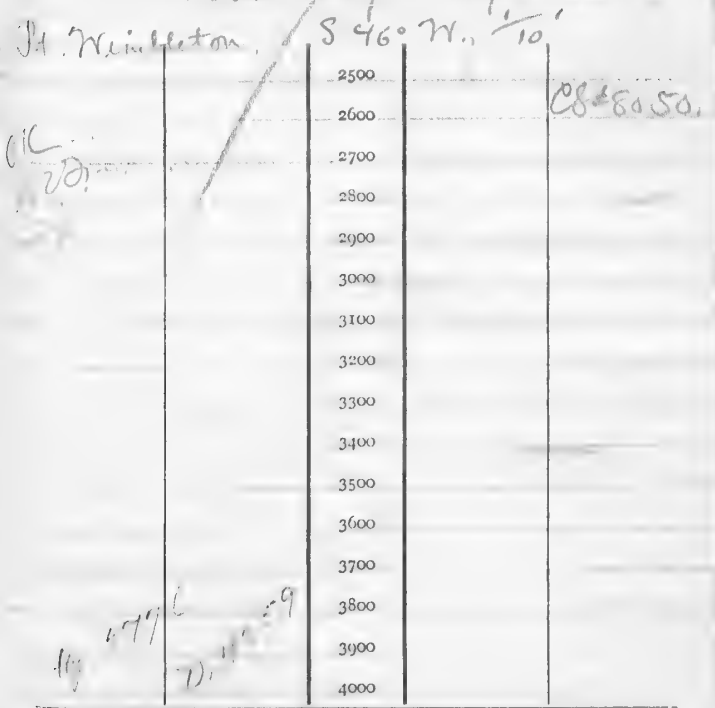

SERIAL TEMPERATURES.

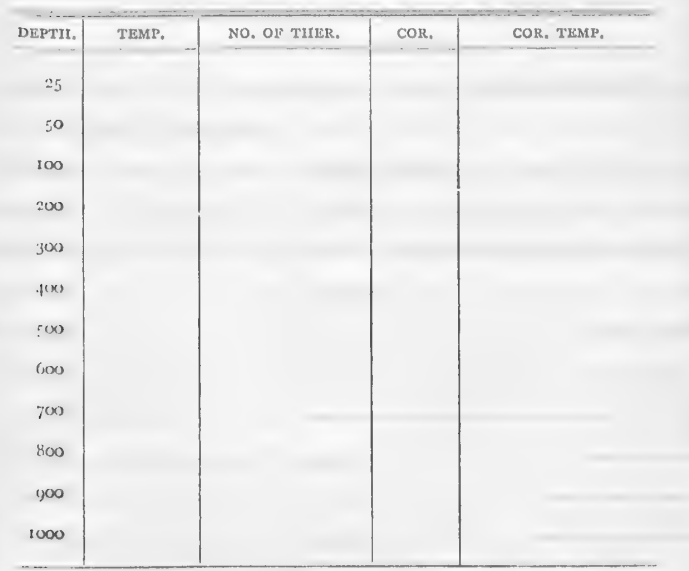

[REMARKS : 
$\therefore$ No. Dis Hn 60 Date Timman Machine, thoet.

Therns io Cor. +1

Depth $2 / \ldots$

Stateor lead 24 Has.

Bottom $R$, as $S_{i}$,

Jiottom temperature $404^{\circ}$

No. of thermometer $98248 \quad$ Cor. 70.2

Corrected temperature $44.2^{\circ} \quad \mathrm{JV}_{1} 6^{\circ} \mathrm{WW}$

Air 59. Surface $5 \%^{\circ}$ Drift $1 / 10^{\circ}$

Trawl onedredge 8:\%

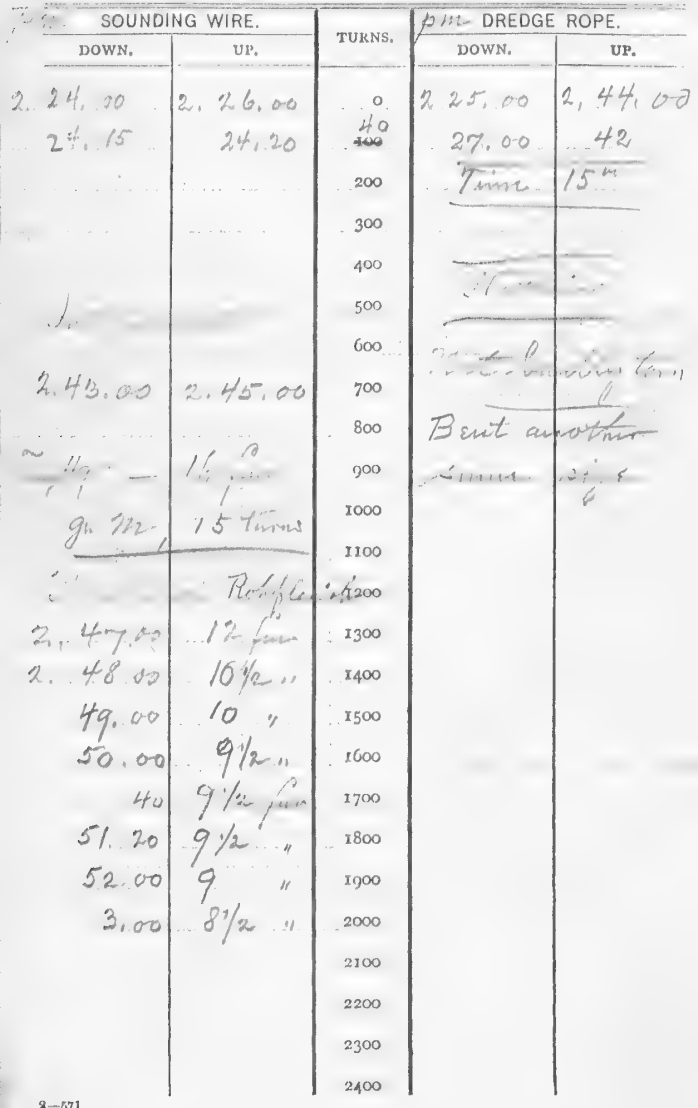


not.

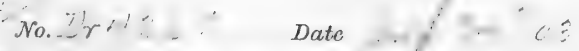

AERHAT. Toel.

Turns ........ Cor. t .... Depth. 10 fan

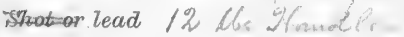

Bottiom gn. A. Aty,

Bottom temperature

No. of therrnometer

Corrected temperature

Cor.

Air 5q Surface 51" Drift $\div 10$

Trawl or dredige 8' Gomen wa before

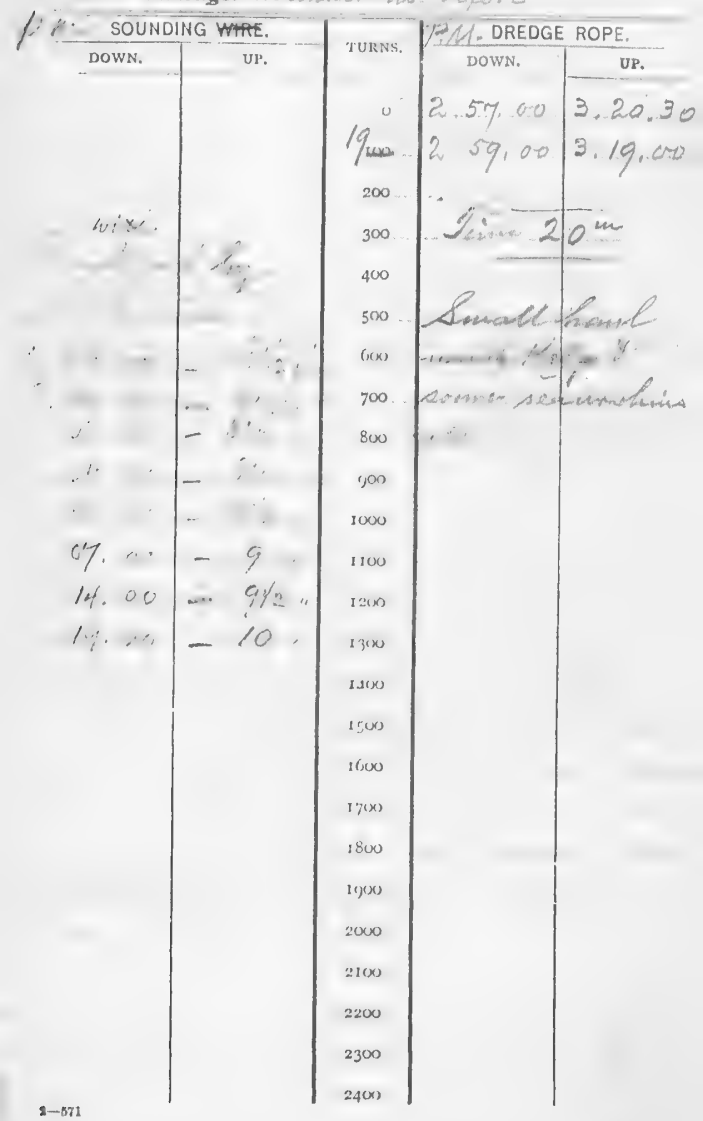




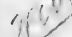

No.Dr $\because 26$ Date

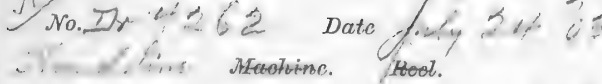

Turns Cor. +

stwator lead /2. if if... \& ...t

Bottom Cus, S. My.

Bottom temperature

No. of therinometer

Cor.

Corrected temperature)

$\{x+y-1$

Air 5 Surface.

Air ... $3 y$ Surface

Depth 9 inis

\section{SOUNDING WIRE:} DOWN.

¿. : $3.0-9 ! 6, \ldots$

$-\quad 3$.

$2-571$
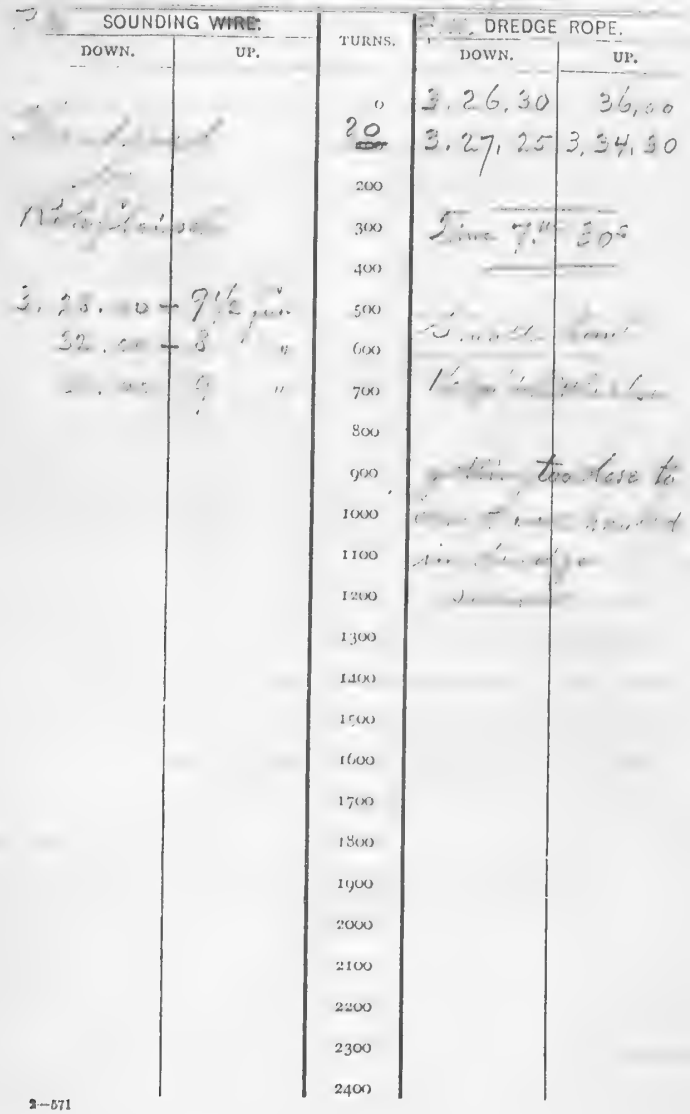

21

Dirt

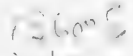

$10^{1}$ 



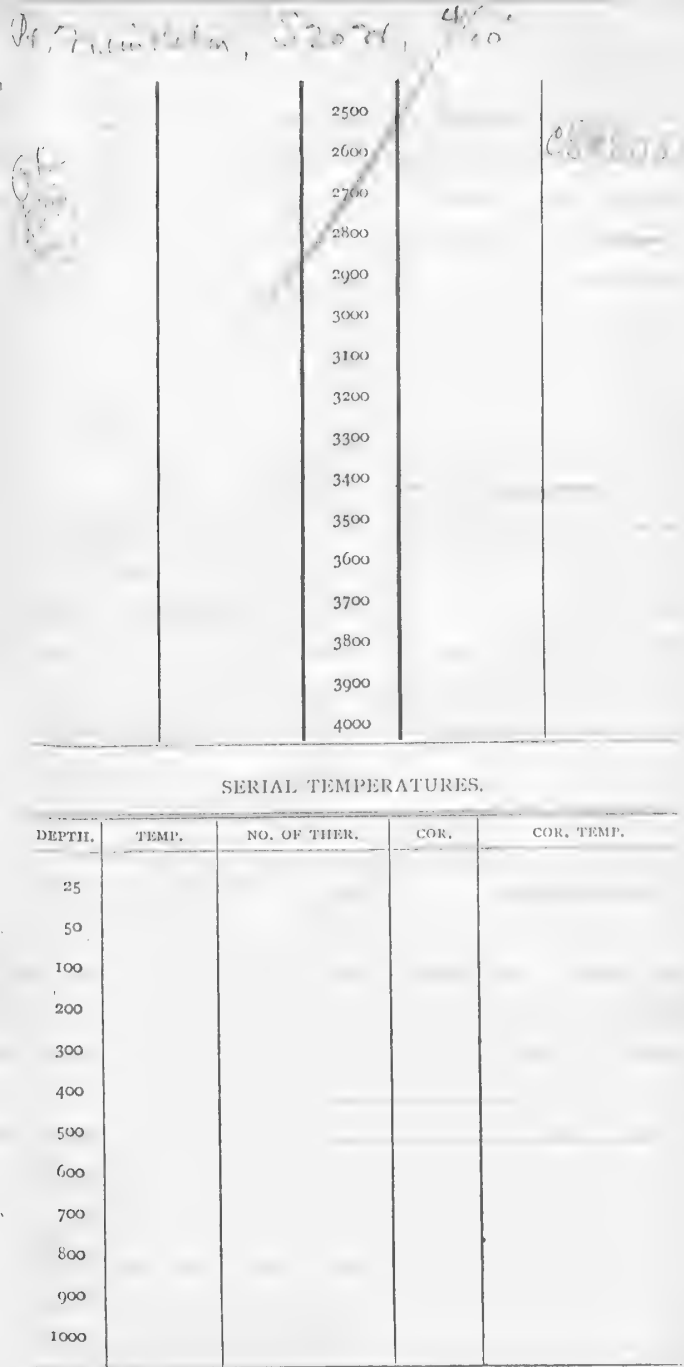

REMARKS : 
nori: I

$1(1)^{12 i}$

No.

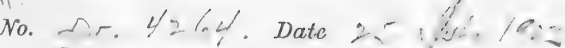

) Trumer Machino Reel.

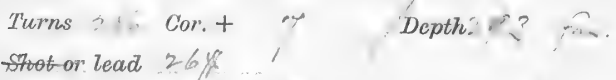

Bottom

Bottom temperature

No. of thermometer Stho thermin. Cor.

Corrected temperature

Air $\sqrt{7}$ Surface 57 Drift $5 / 10$

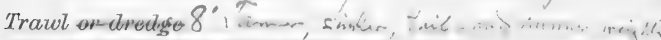
1,... SOUNDING WIRE.$$
2
$$

34.20

$\$ 0.20$

$2.41 \cdot 40$

UP. TURNS.

DREDGE ROPE.

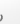
- . एi. un

$\int 4 e_{1}^{2}, 25$ ?. $4: \ldots$

. Clirk, $\quad \therefore 1, \therefore$. $1 \therefore=\because ;$ Saener $\therefore t, 5, \therefore 1,1,1, \cdots$

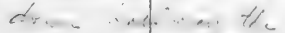

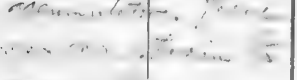
.. . . $2-671$

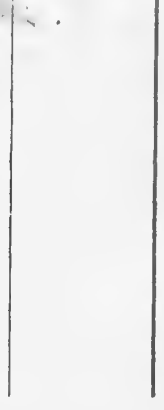

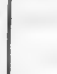$$
2000
$$

200

$3(x)$

400

$5(x)$

$(r)$

700

800

g(x)

suov 

son.

No.<smiles>C1CCC2CCCC2C1</smiles>

NMCNE... Date.

Turve 198 cor. +6

Reel.

stootor lead 2 of the

Depth 204 Am.

Botiom gn $(v)$, in 6 , $5 / 2$

Bottom temperature 1420

No. of thermometer ...98.248 Cor.

Corrected temporature

Air ... 53 Surface $53^{\circ}$. Drift

Frototur drectero

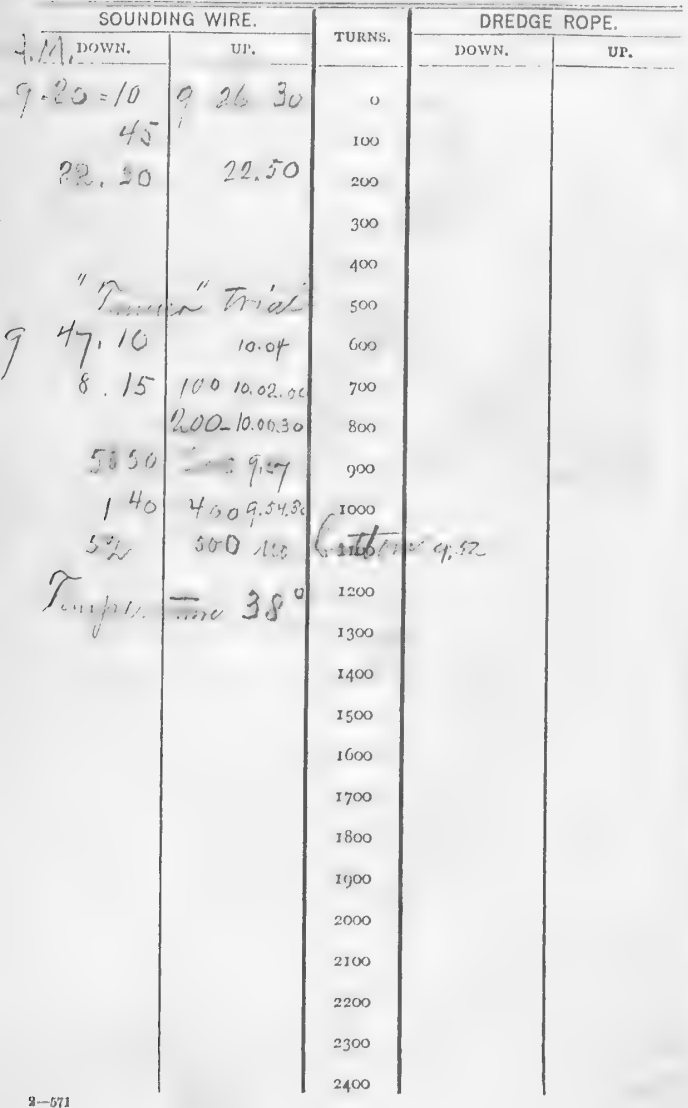



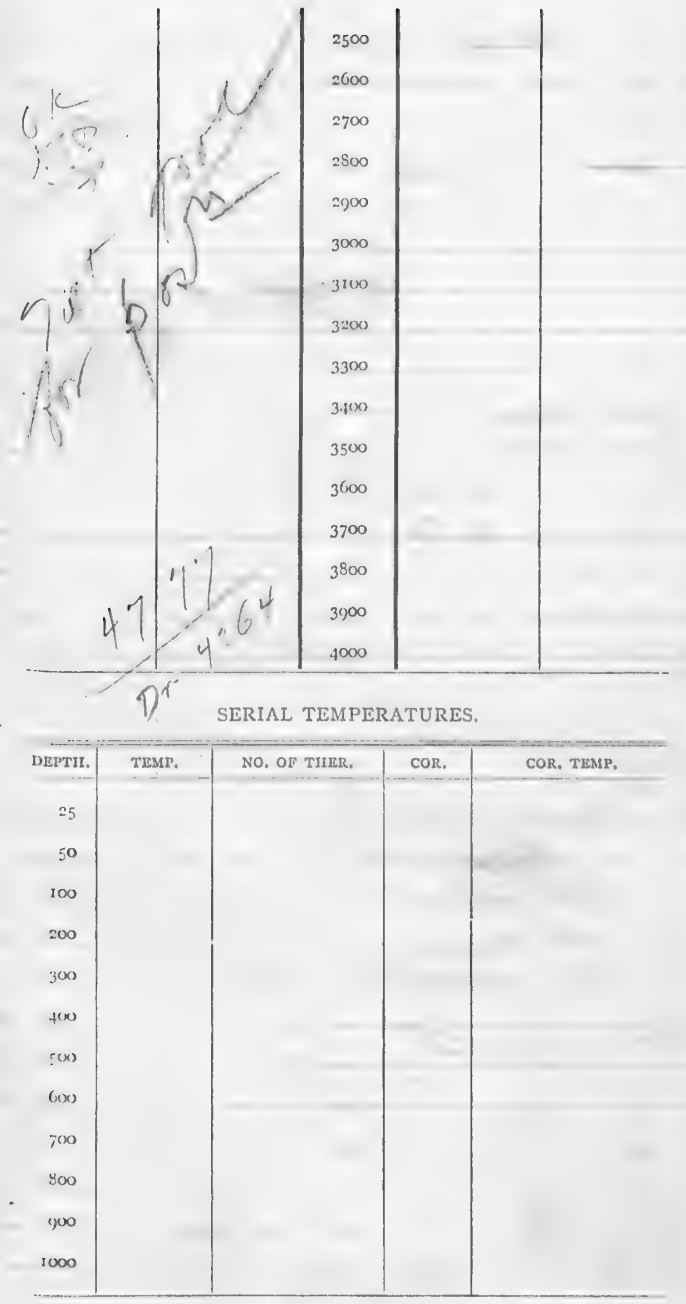

REMARKS : 
No $7 \div 26 \sqrt{\text { Date }}$

R

$y^{m}$

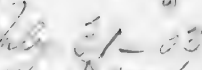

Jig Machine.

Thins 541 Cor. +40

Depth 590

Shat ar lead $36 \mathrm{fb}$

Bottom

Bottom temperature $38 \%$

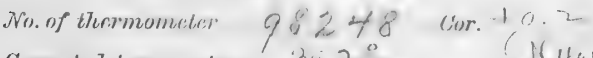

Corrected temperature $3 \% .2^{\circ} ? \times 44^{\circ}$ ?

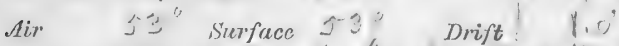

Trawl

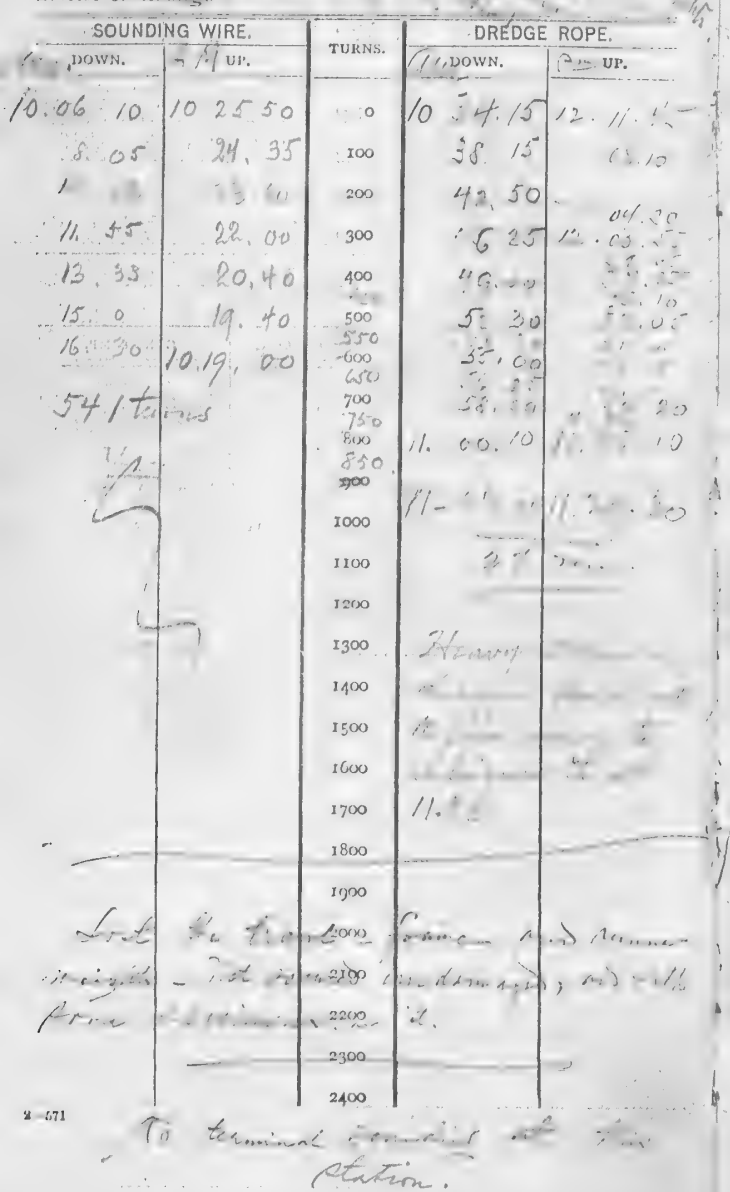


obi setkal sound,

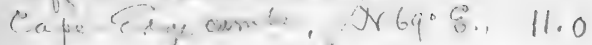

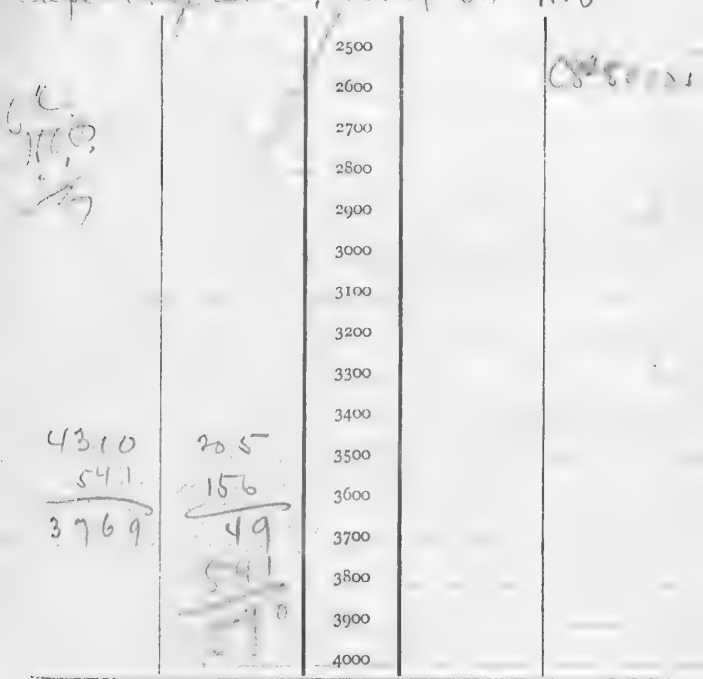

SERIAL TEMPERATURES.

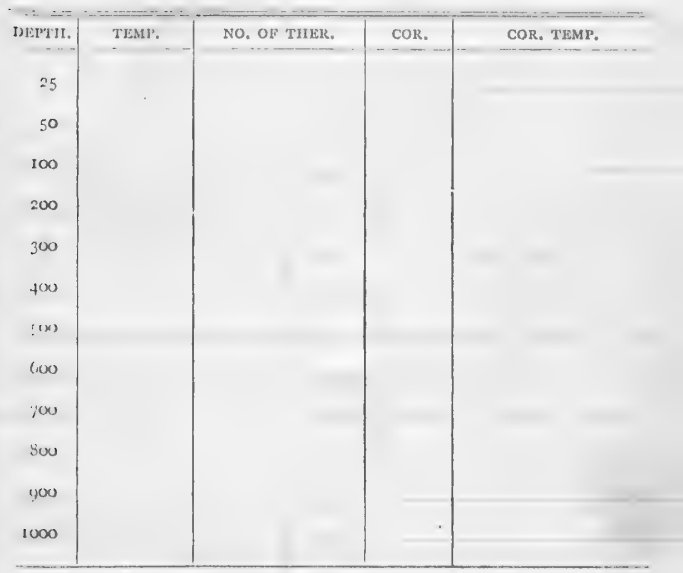

REMARKS: 
(n)

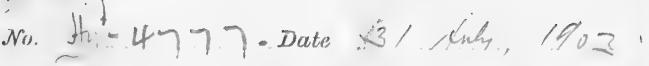
Vanuer Machine : Recl.

Turns of Cor.t \& Depth ? - in.

Stotor lead. $26 \%$

Bottom a. S. wh. S..P.

Bottom templerature. ' 42.1

No. of thermometer 98248 . Cor. $+0,2$

Corrected temperature 42,30

Air. $62^{\circ}$ Surface $\sqrt{-6} 6^{\circ}$ Drift

Iroustor alverlige

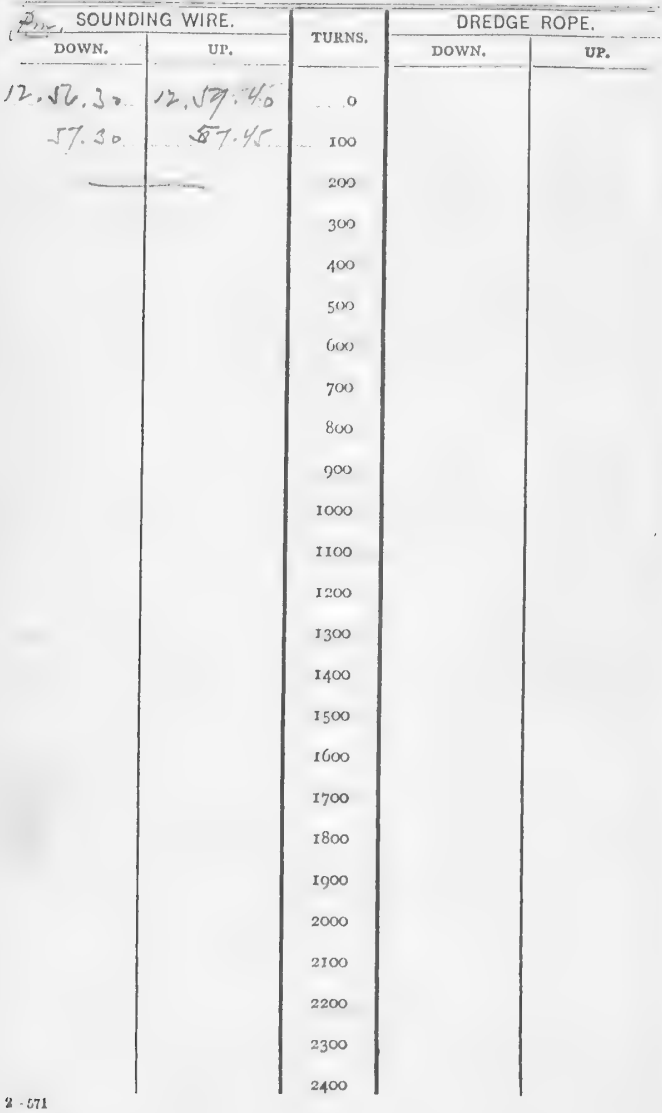




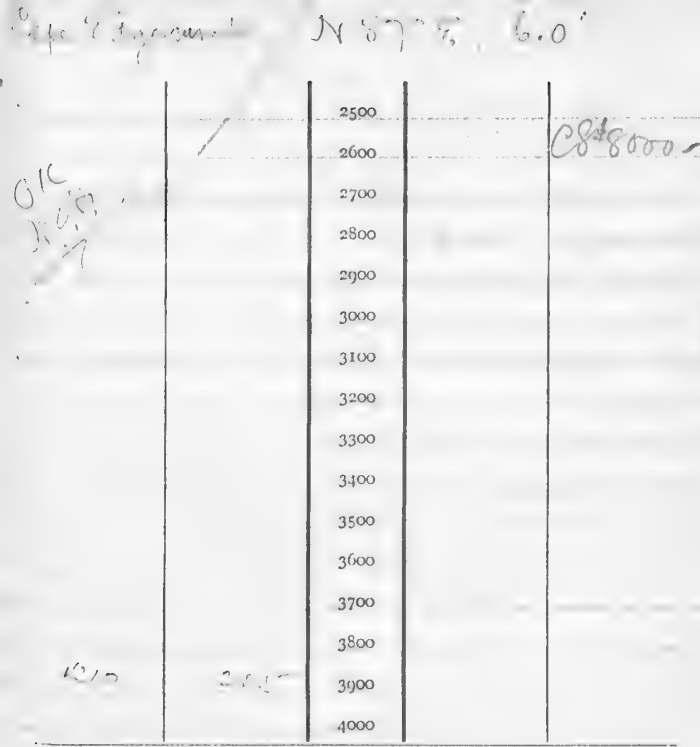

SERIAL TEMPERATURES.

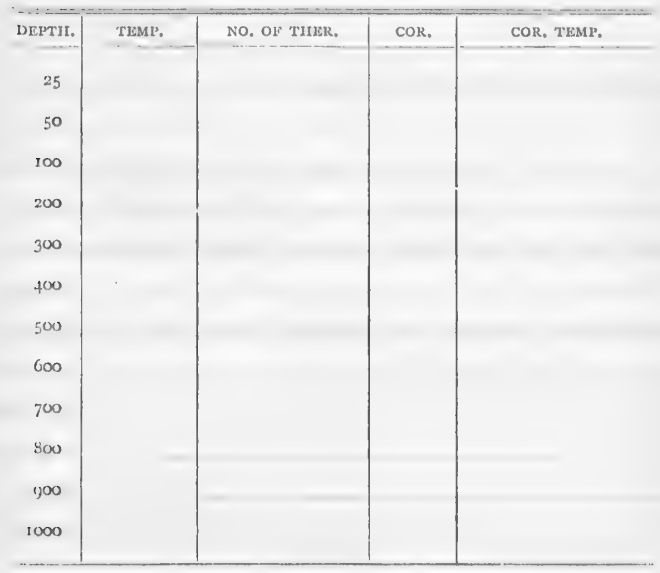

REMARKS : 
507. $k i 1$

Q $\rho^{i}$

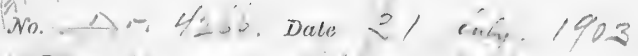

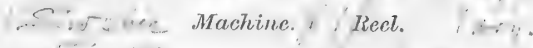

Therns $3 / 7 \mathrm{Cor}+32$ Depth $34 \mathrm{G}$ Kn.

Botitom

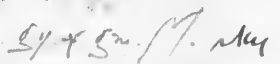

Bottom temperature

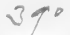

No. of thermorneter

$$
98=28.0 \text { Cor. }-10.2
$$

Corrected temperalure

Air $62^{\circ} \quad$ Sierfuce $\sqrt{6} 6^{\circ}$ Drift $7 /{ }_{1}^{\prime}$

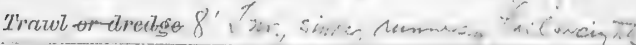
6... SOUNDING WIRE. DREDGE ROPE.

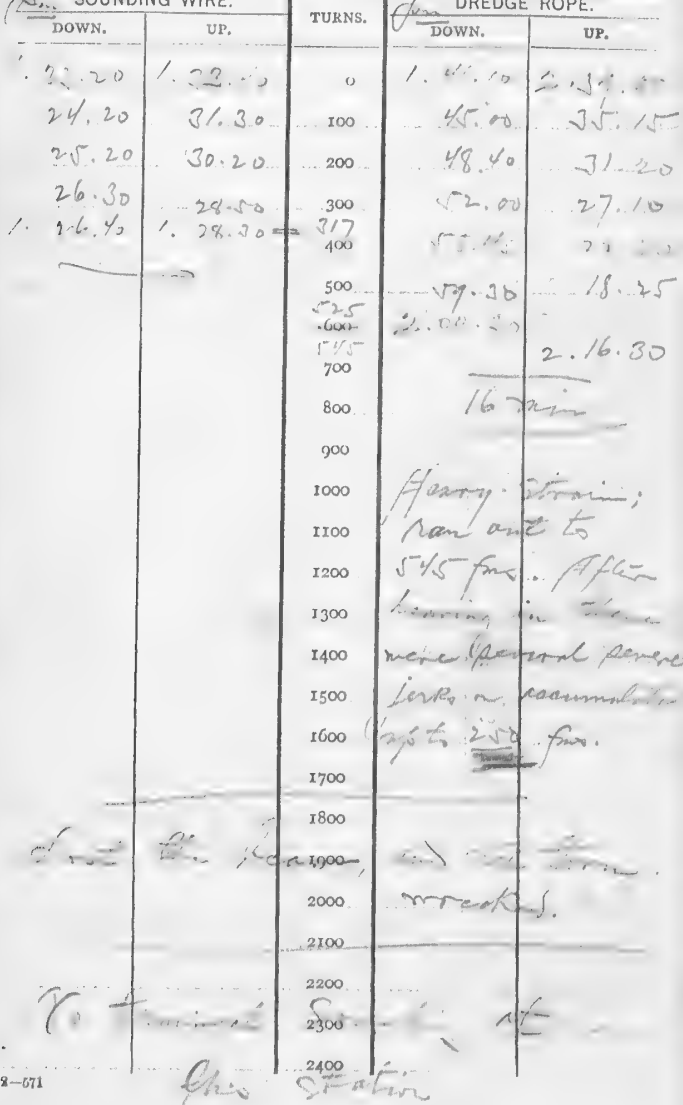




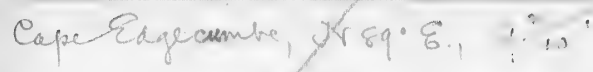

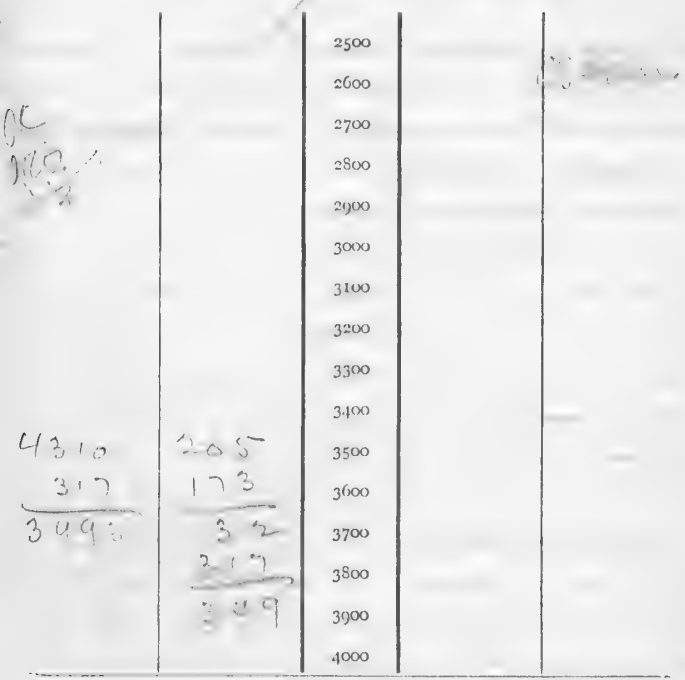

SERIAL TEMPERATURES.

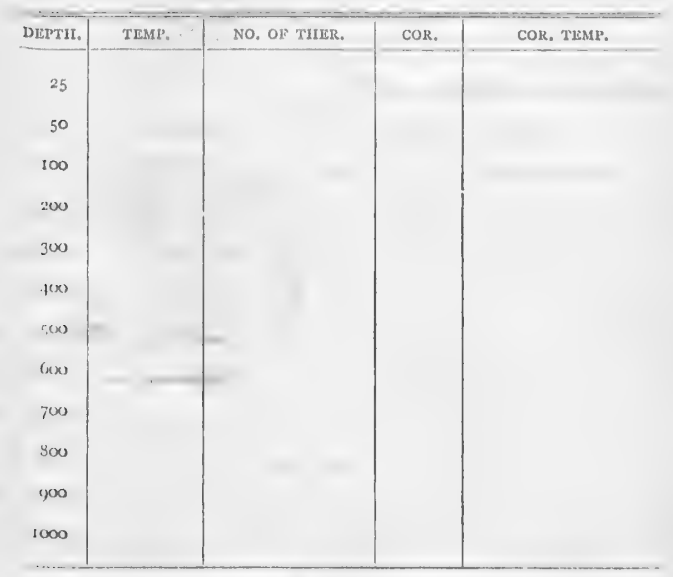

Tret wreaced hemarks: $-\lambda$ temis is

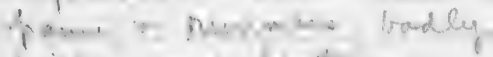
I. in: $+\cdots$. 


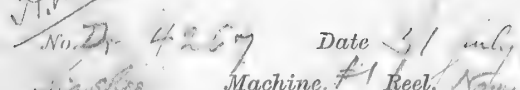

-itsue Machine f' keel. Natu.

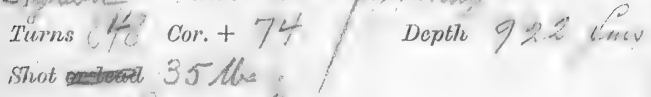

\section{Botiom st.}

I3ottom temperature $36.1^{\circ}$

Non of thermometer. 982 ? Cor. +0.1

Corrected temperature $36.2^{\circ}$ ( J161" Air. $5 y^{\circ}$ Surface 58 Drift $) ~ 1 / 10$ '

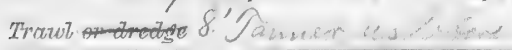

\begin{tabular}{|c|c|c|c|c|}
\hline \multicolumn{2}{|c|}{ SOUNDING WIRE.P $/ M$} & \multirow{2}{*}{ TURNs. } & \multicolumn{2}{|c|}{ DREDGE ROPE, } \\
\hline Down. & vi. & & nown. & vi. \\
\hline$\therefore: 0$ & 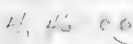 & 。 & 45100 & $16,4 \mathrm{~d}$ \\
\hline$\therefore 15$ & $\therefore 20$ & 1100 & 54,45 & 44.00 \\
\hline 710 & $4+500$ & 200 & $5 \% \quad 36$ & $\therefore 9.0$ \\
\hline$?$ & 12.0 .5 & 300 & {$\left[\begin{array}{lll}5 & 0.1 & 1\end{array}\right.$} & -640 \\
\hline$\hat{i} \hat{\circ}$ & 12,05 & 400 & 0.: :0 & 33.10 \\
\hline $30 . \infty$ & 41.05 & 500 & 0830 & .50 \\
\hline $1 \%$ & $\because 0 \mathrm{cn}$ & $6 \infty 0$ & 11.4 & $\therefore 10$ \\
\hline$\because \quad \because$ & $4 \div 8<0$ & 700 & 14. $\approx$ & 20.20 \\
\hline $3:-$ & .27 .02 & 800 & $\therefore 80$ & $18 .=0$ \\
\hline downi sis & $8 t$ & 2000 & $a$. & 13,30 \\
\hline & 4.35 .58 & 1000 & $\because 1$ & \\
\hline & & 1200 & $\therefore:$ & $\begin{array}{lll}6 & 0 & 10\end{array}$ \\
\hline & & 1300 & $28 . ?$ & 56.10 \\
\hline & & 1400 & $=39.4$ & 5.51 .30 \\
\hline & & 1500 & & $\ldots+\infty$ \\
\hline & & 1000 & 204 & 1 \\
\hline & & $\begin{array}{l}1700 \\
1800\end{array}$ & Simall & haw. \\
\hline & & 1900 & & \\
\hline & 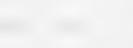 & 2000 & 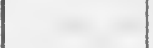 & \\
\hline & & 2100 & & \\
\hline & & $\begin{array}{l}2200 \\
2300\end{array}$ & & \\
\hline & & & & \\
\hline
\end{tabular}




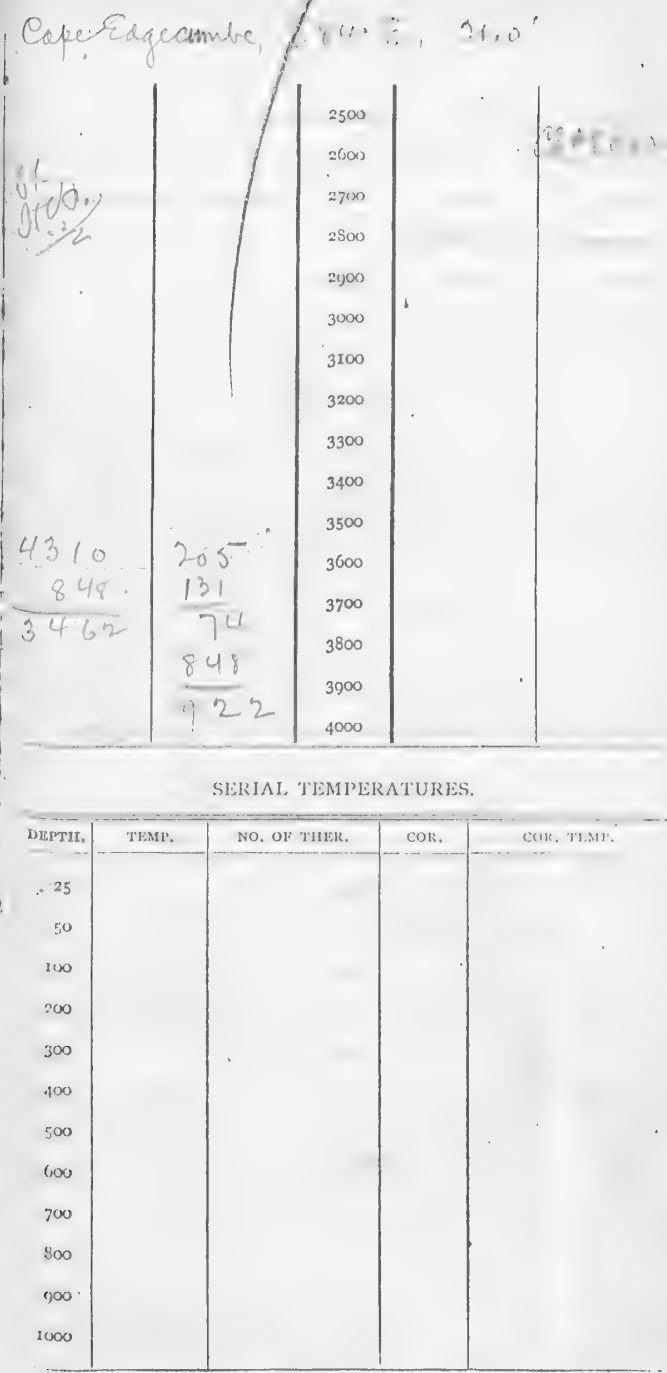

REMARKS: 
कr -

$(1,1)^{6}$

No. $1,-4268$ Date $3,190=$ 1. Trune Machine. Reel.

Turns 16 Cor t 0 .... Depth 16 frim. Hot or lead 26 사

Bottiom.

$$
\text { sys }
$$

St $t_{n}$.

Botiom temperature. $\sqrt{10}$

No. of thermometer बQ2 24 Cor. $-0,1$

Corrected teinperature Air $\sqrt{ } 7^{\circ}$ Surface 50.40

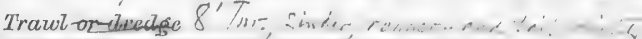

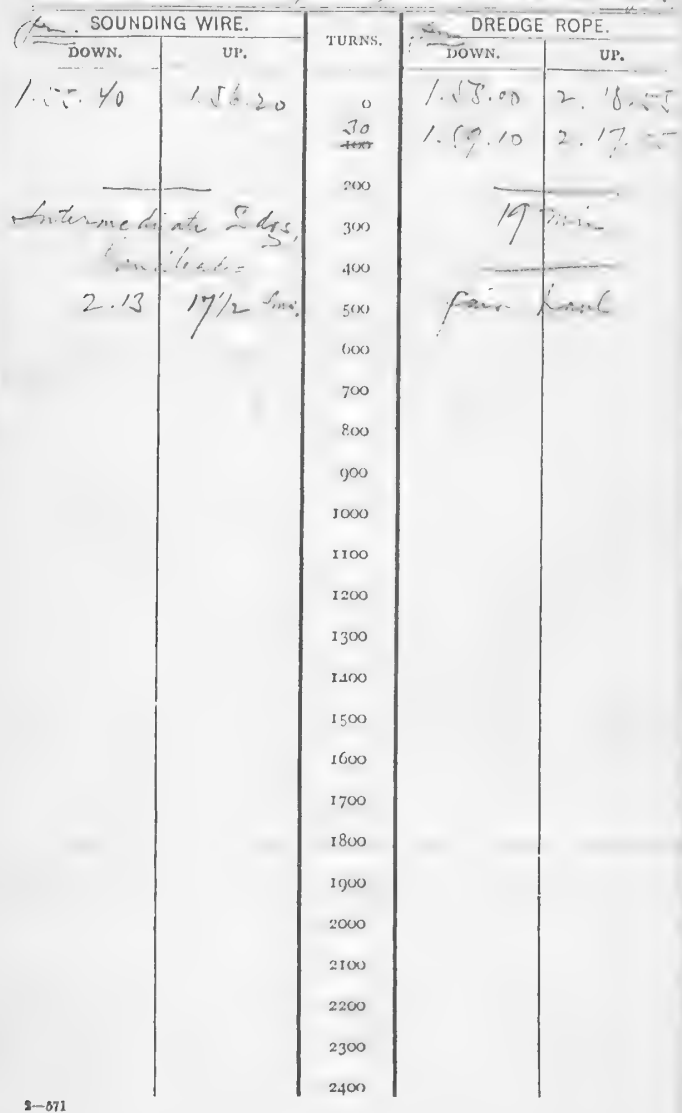




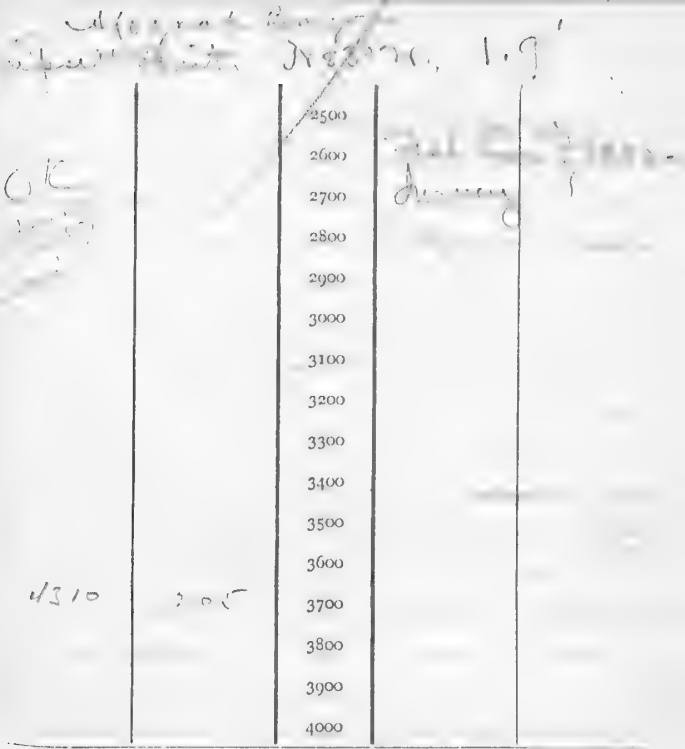

SERIAL TEMPERATURES.

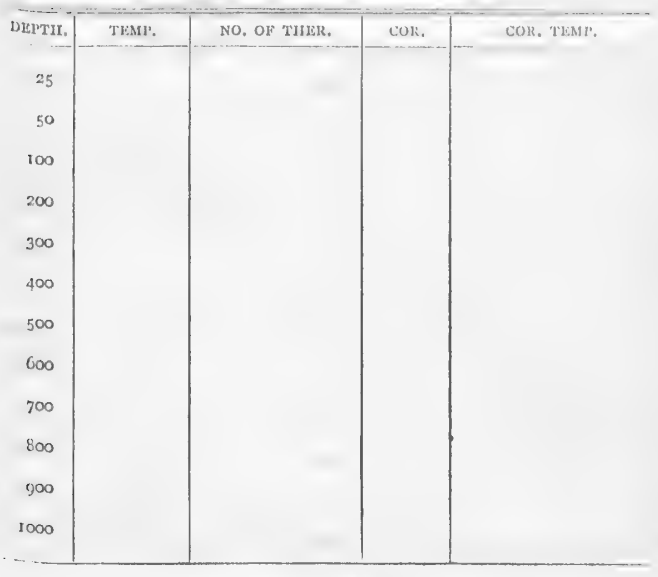

REMARKS : 
$\left|r^{i}\right| \sigma^{2} \mid$

No.

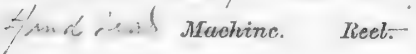

'Turns ........ Cor. +

Shotweread /Y X,

Bottom K, K.

Bottom temperature

No. of thermoineter.

ro then. Cor.

Corrected temperature

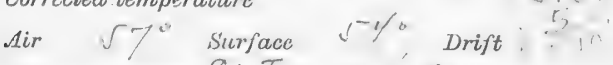

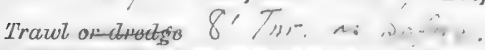
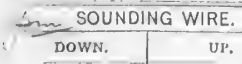

UP.

2. 24.30

$\left(14^{\prime} / 2 \sin ^{\prime}\right)$

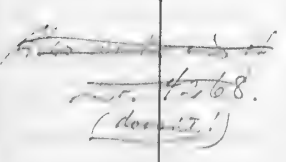

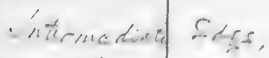
$\therefore+1 .+1=$

2. ? $2=$

2. $3 \cdots$

7. 114

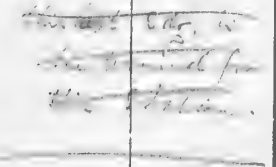

2.17
3.04

126

14 ,

8. 871

$11 \hat{x} \therefore$

$17 \%$

13

1200

1300

1400

I 500

I600

1700

1800

1000

2000

2100

2200

2300

2400
Depth $14 \%$ fris. 


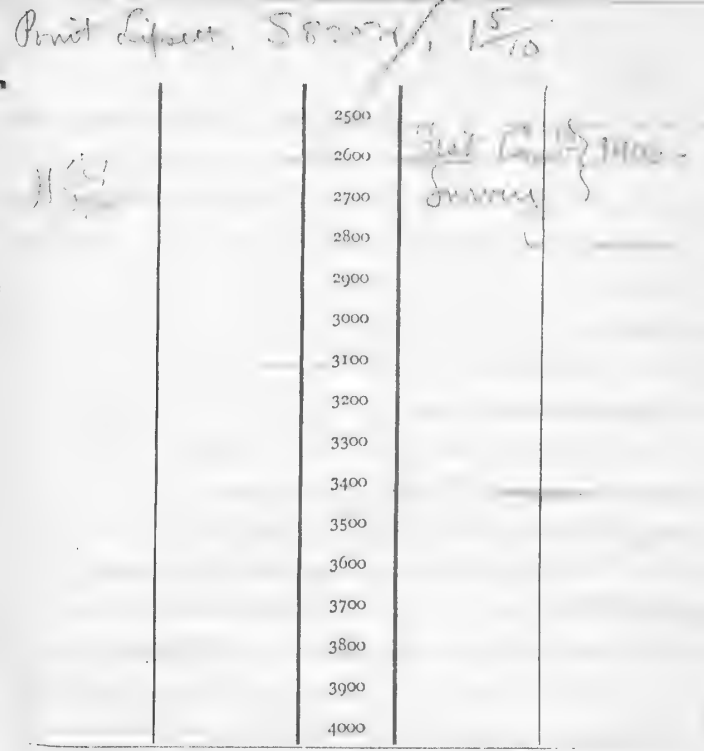

SERIAL TIMPERATURES.

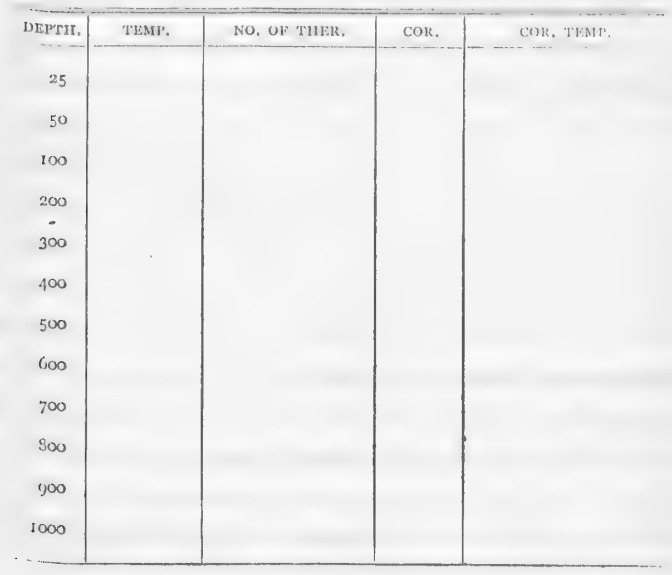

REMARKS : 


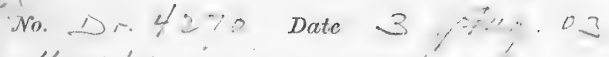

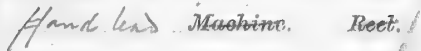
Turns
Cor. +
Shotas lead
14.45
Depth

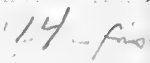

Bottom

Bottom temperature

No. of therrnometer.

$\zeta$ i ín

Corrected temperature

Air $\sqrt{6} 6^{\circ}$ Surface $\sqrt{3} 3^{\circ}$. Drift $1 / 2^{\prime}$

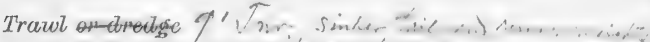

SOUNDING WIRE.

ur.
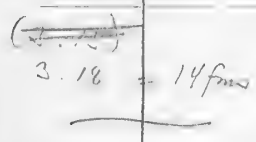

vivinatificis

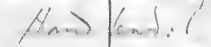

3. 22

3.28

i. 34

J $/ 0$

$(x+i)$

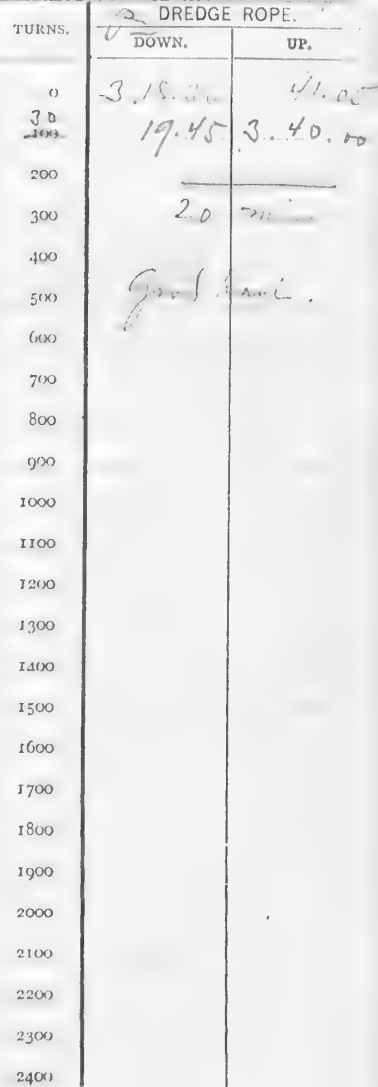




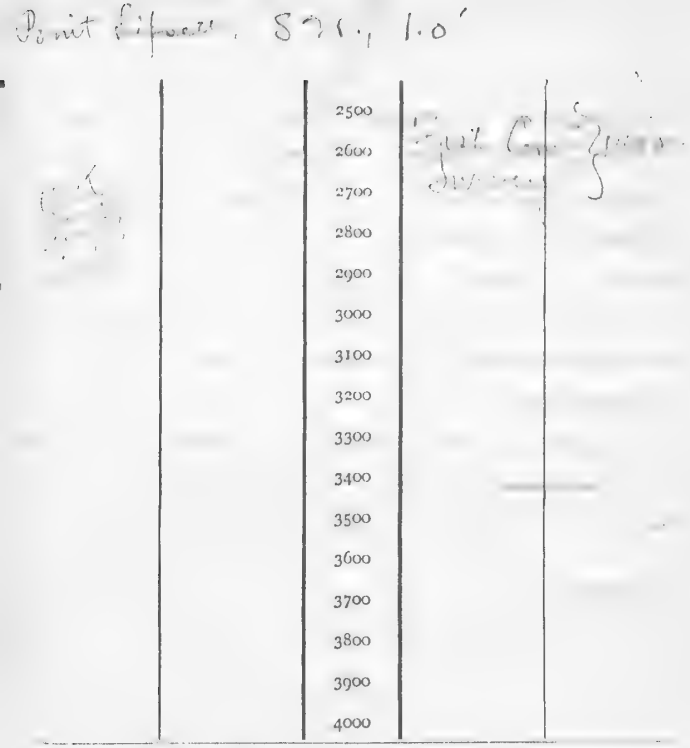

SERIAL TEMPERATURES,

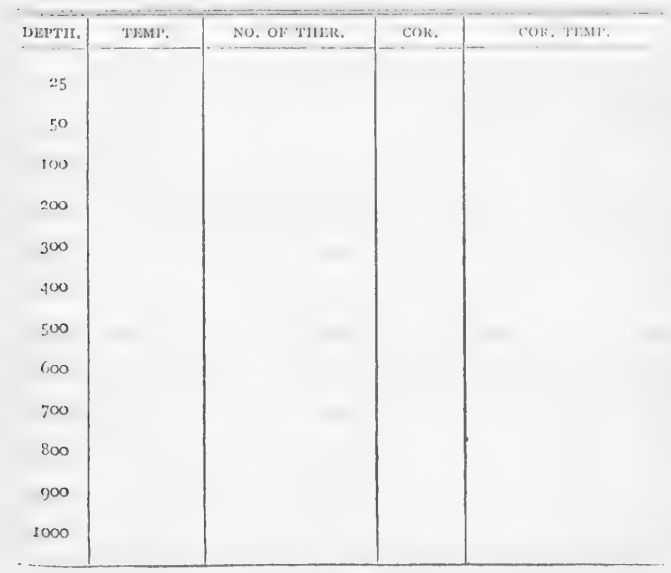

REMARKS : 
No. I, 4 27 , Date -

J Hun $L$ end Machine. Reel.
Turns.
Cor. +
Stot or lead
$14 \times 11$
Bottom
$\lambda_{1}-\alpha$.

Bottom temperature.

No. of thermometer.

$\left\{z_{0}\right.$ thensas, Cor.

Corrected temperature

Air $\sqrt{1} .^{\circ}$ Surface $\sqrt{3^{\circ}}$ Drift $], \ldots$

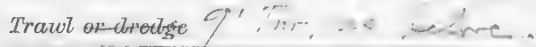

$\checkmark$... SOUNDING WIRE.

$$
\text { DOWN. }
$$

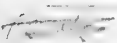

?. 40

5

$\therefore+$

3

(

UP.

, .

$\therefore$

10 $4.00,00$ $4.06,30$ $4.13,00$

1

TURNS. $1 \frac{\text { D. DREDGE ROPE. }}{\text { DOWN. }}$ (3) 3. $\because \cdots, \overline{\because 11,00}$ $7 n y$. 1112 2- 671 

nor? (1)

ye

No. ㄴ

$\because 6 \cdots$

Date $\checkmark$ Machine. Reel.

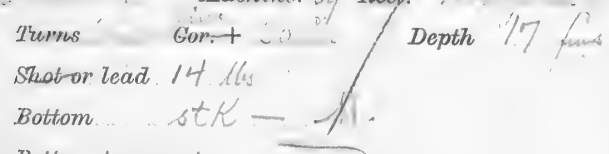

Bottom temperature

No. of thermometer twot usied

Cor.

Corrected temperature

Air 52: Surface 5\%०

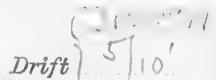

Trawl or-diredge

$D$ in SOUNDING WIRE.

$$
\frac{P \text { SOUNDING WIRE. }}{11}
$$
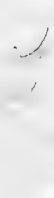

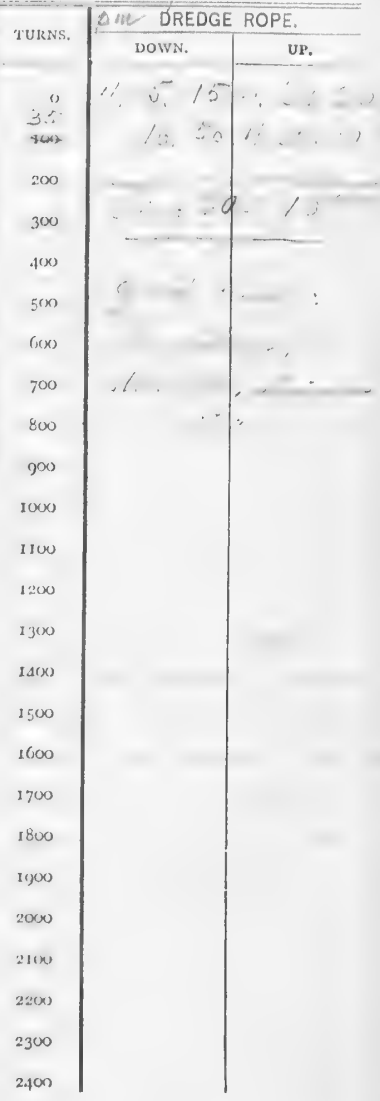




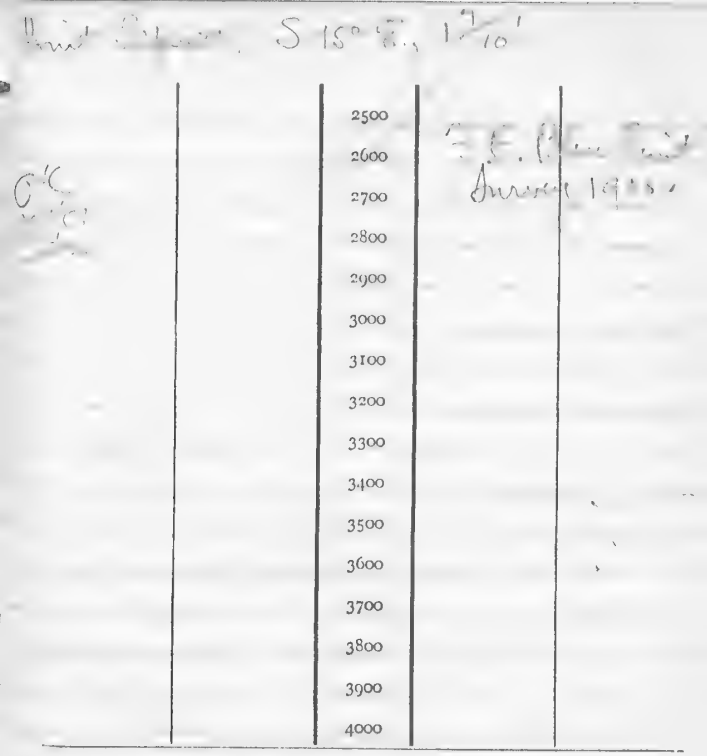

SERIAL TEMPERATURES.

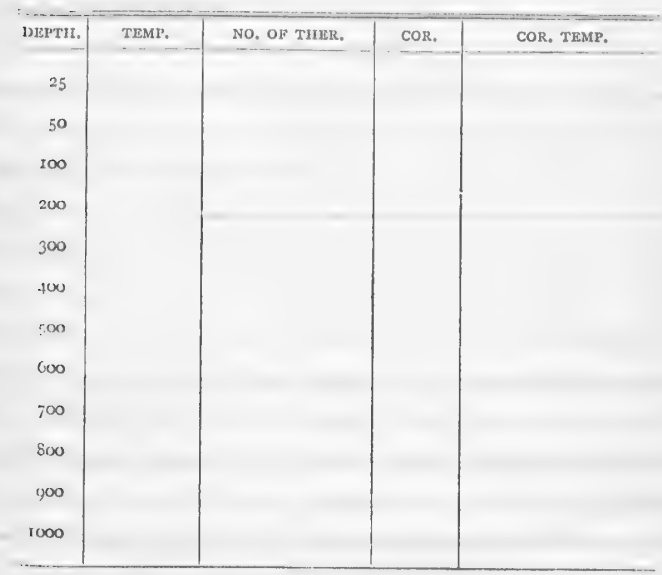

REMARKS : 
607.

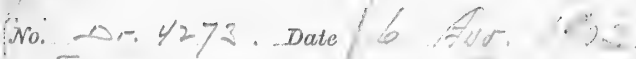

$1 \sqrt{\text { rmmer Machine }}$ Reel.

Turns 35 Cor. + Depth 36 fin.

Stuobor lead 26,4

Botiom andin. huls.

Bottom temperature

cartiond roma.

Non of thormometer $\$ 8248$ Cor.

Corrected temperature

Air $\sqrt{3}$ Surface $5 /$ Drift $8 / 10^{\prime}$

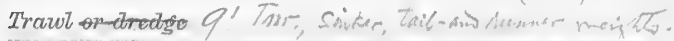

SOUNDING WIRE.

DOWN, |

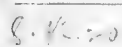

8. 42,10

soth lean stron Sritiva - at 35 twrns wine Ale th the reet, thinks, and $\sin \alpha \beta \cos \alpha$. Lost a 26 is lecif thermons.

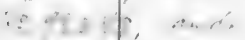

$\therefore 6+x \int_{1}^{\prime} 5 \therefore \ldots$ $\therefore \quad l=2(r, \ldots)$

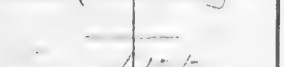

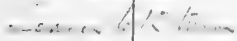

$4+1,0$,

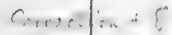

$\therefore \div 2$

$\therefore \ldots$

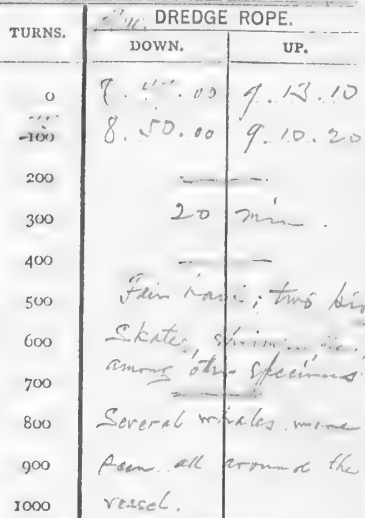

TURNS.

$\therefore / \%$ DREDGE ROPE. \begin{tabular}{l|l} 
DOWN. UP. \\
\hline
\end{tabular}

0 7.4.110 $\% .5 .10$

$-1(x)$

200

300

400

500

600

700

800

900

1000

t100

1200

1300

1400

I500

1600

$\times 700$

I 800

Igoo

2000

2100

2200

2300

2400 


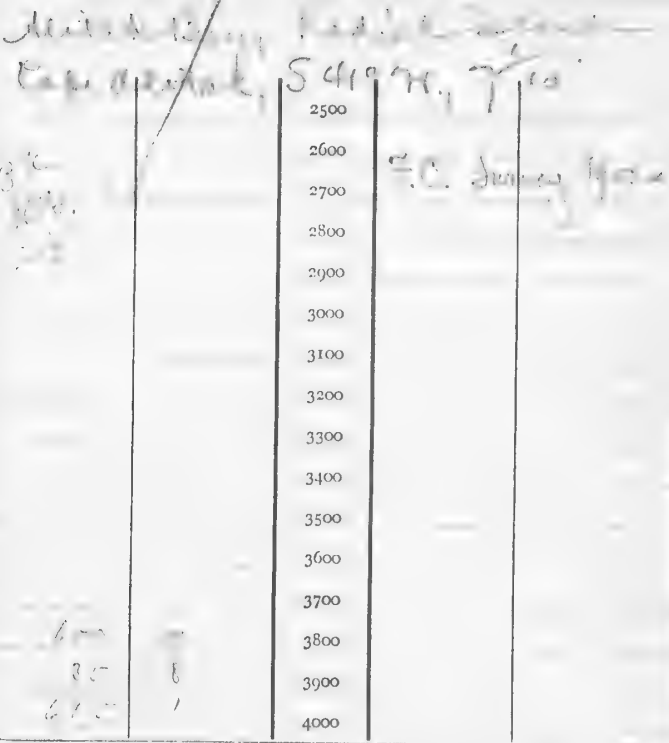

SERIAL TEMPERATURES.

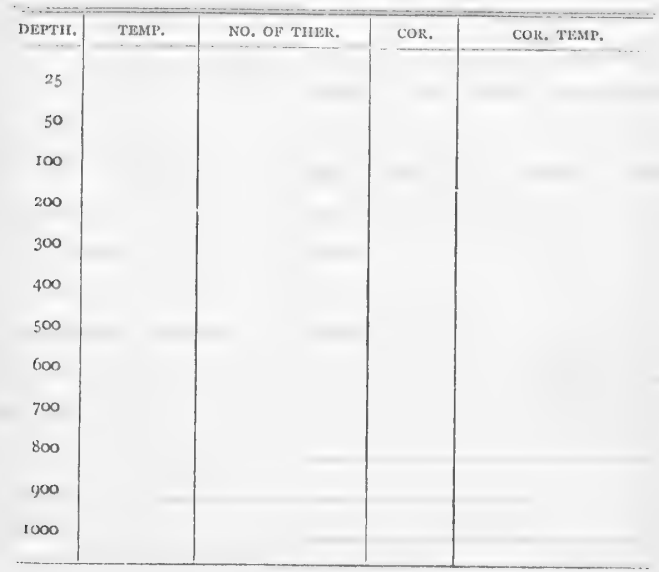

REMARKS : 
[n, $1 ; 11>$

No.

4274 Date

6 HNo.

$190=$

- l'Mins Machine. Reel.

Turns $? 7 \mathrm{Cor}$.

2 Depth

Sthot-or lead $26 \%$

Bottom qu. M. fu. S.

Bottom temperature

No.of thermometer / Vo theras.

Cor.

Corrected temperature

Air

Sierface

Drift

$6 /, 1$

Trawl ordutige of' Tum as hedrue

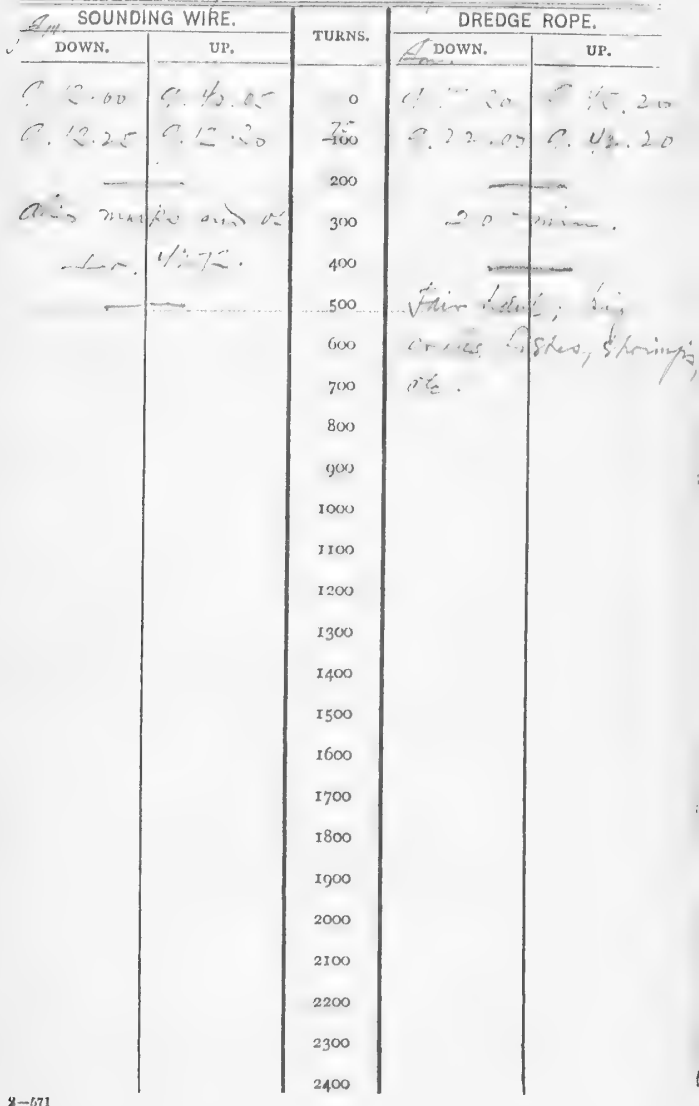




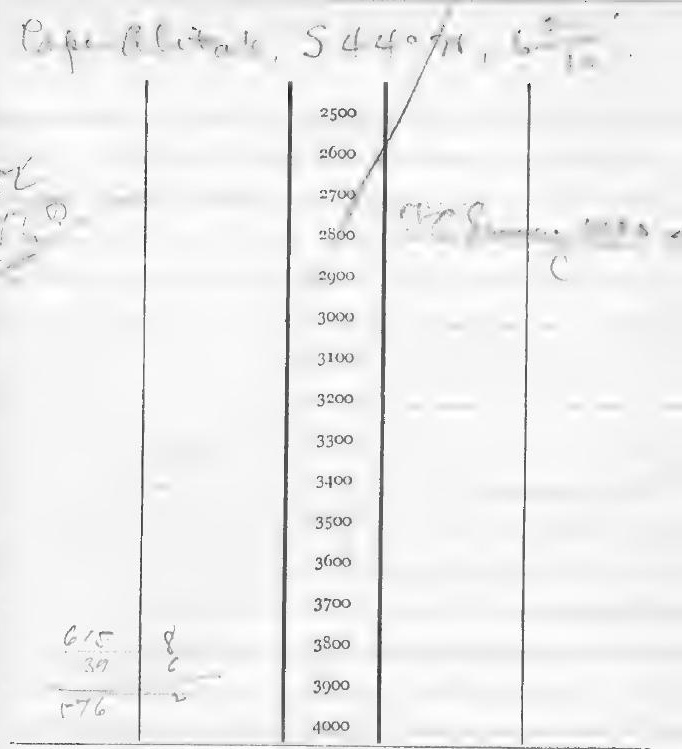

SERIAL TEMPLRATURES.

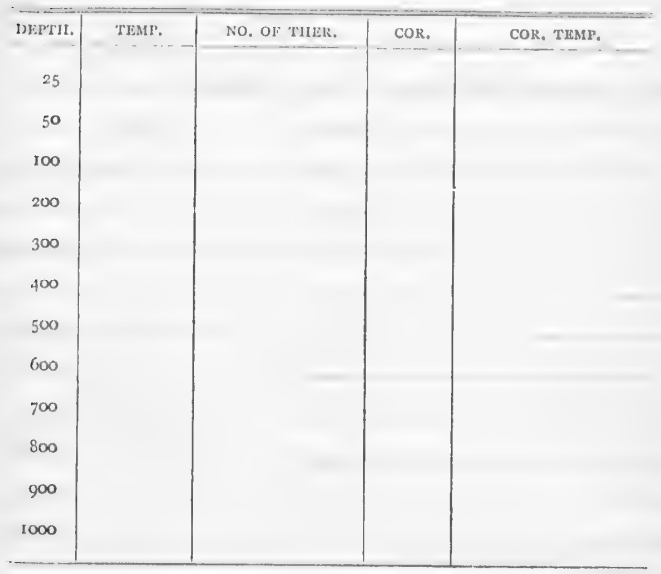

REMARKS : 

Arilil'

No. 4 Y Y778. Date $\checkmark$ amer Machine. Reel.

Turns 35 Cor. +11 Depth 36 fins.

lead $26 \%$

Bottom gn.

Bottom temperature 44.2

No. of thormometer

Corrected temperature

$9824 \%$ cor. to. 2

Air $\sqrt{2}$, Storface $\sqrt{ } /$. Drift

\section{Thedut-or-dredge}

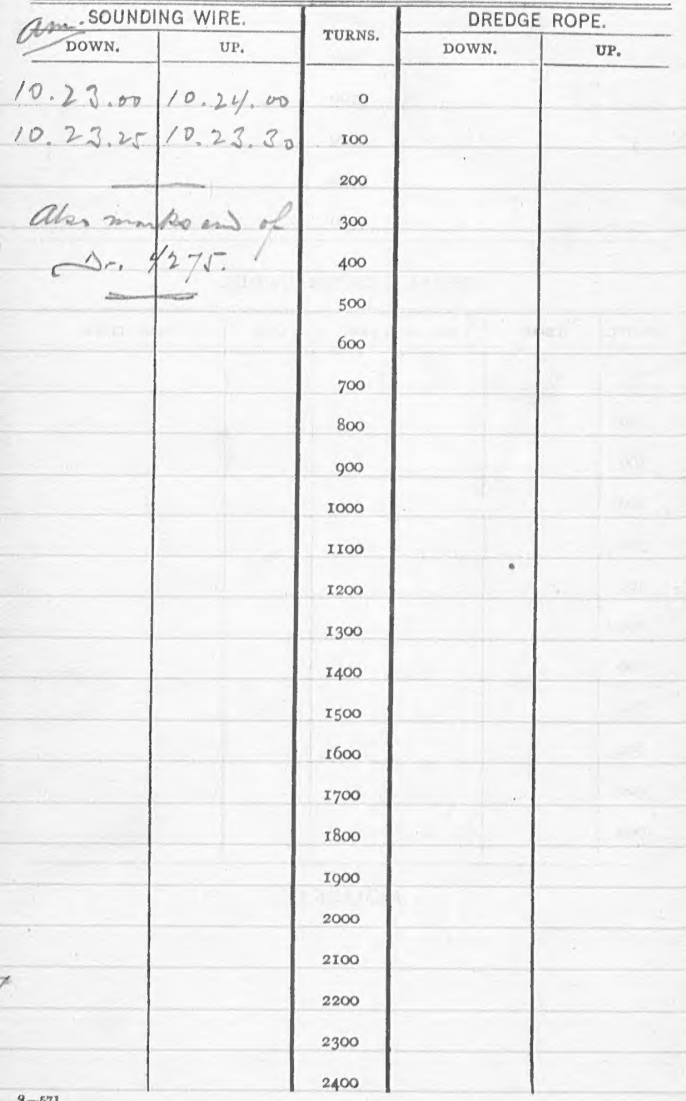




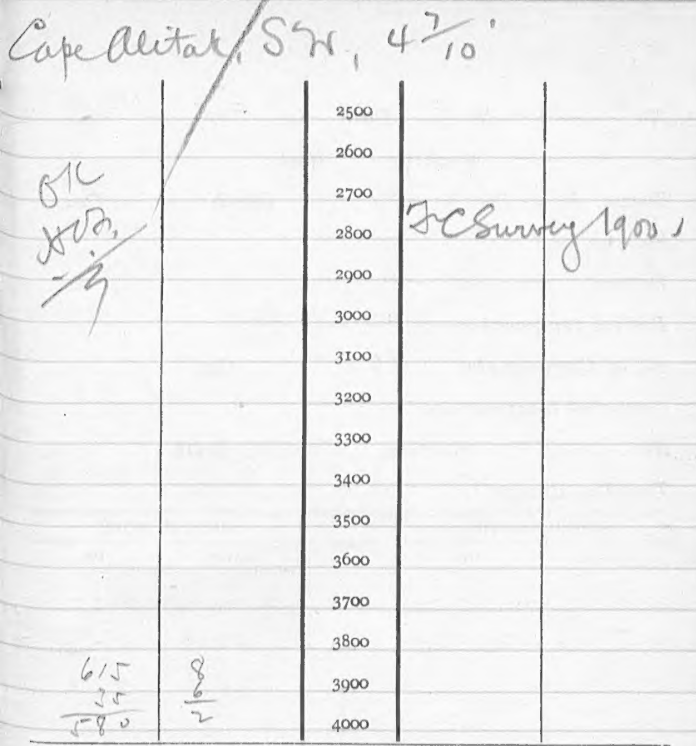

SERIAL TEMPERATURES.

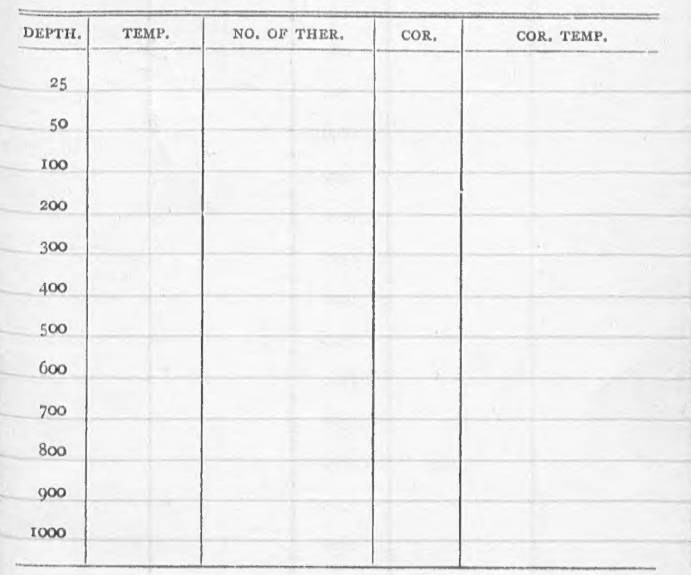

REMARKS : 
507. :

a. 1 th $^{2}$

No. Nor. 4276 . Date 6 7vi, / 1903. J Noms Machine Reel.

Turns 21 Cor. + Depth 22 fors. strot or lead $26 \%$

Bottom fine. S. N.

Bottom temperature T. divint tris

No. of thormometer 982.7 Cor.

Corrected temperature

Air $\sqrt{5} 5^{\circ}$ Storface $52^{\circ}$ Drift $8 / 10^{\circ}$

Trawt or aredge g' Trenur as Leforem.

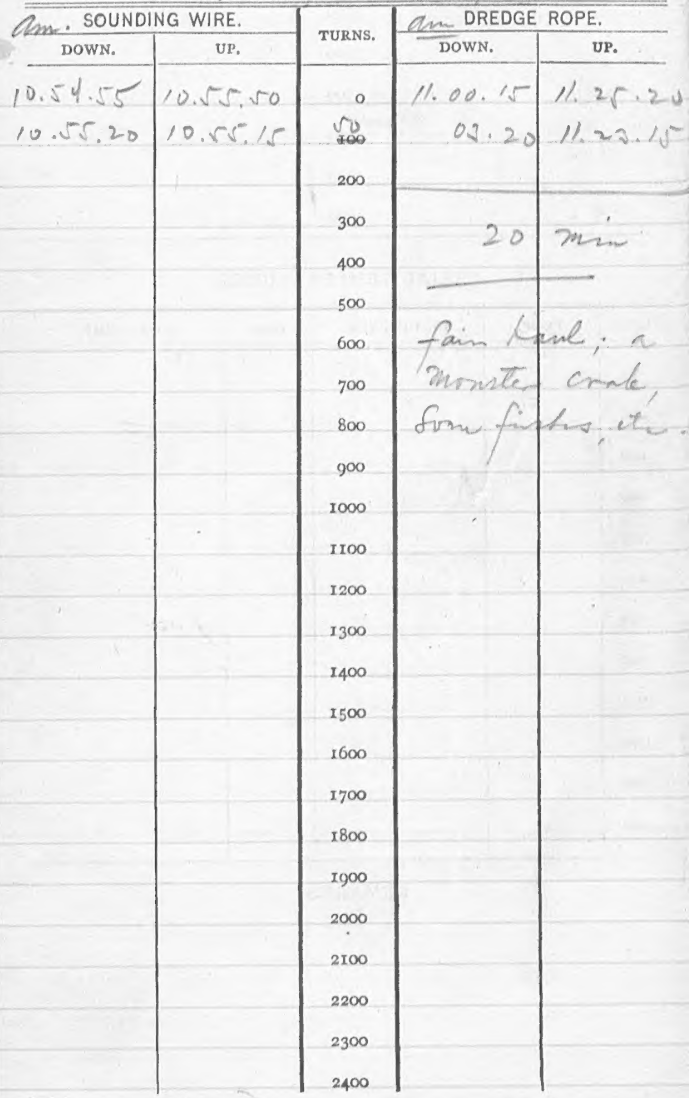




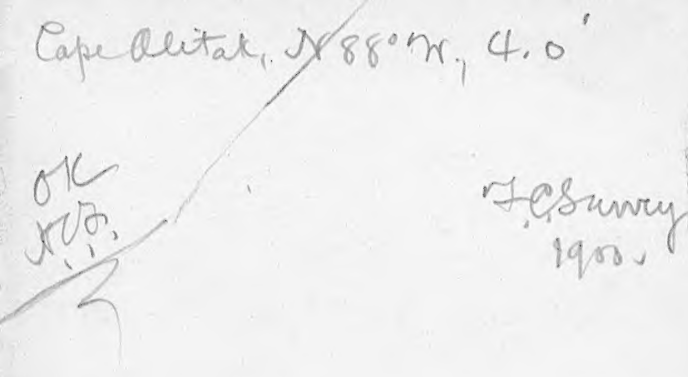

$$
\begin{array}{r}
65-8 \\
21-7 \\
594
\end{array}
$$

